\title{
New Frequency-Dependent Trigonometric Interpolation Functions for the Dynamic Finite Element Analysis of Thin Rectangular Plates
}

\author{
Supun Jayasinghe $\mathbb{D}$ and Seyed M. Hashemi \\ Department of Aerospace Engineering, Ryerson University, Toronto, ON, Canada \\ Correspondence should be addressed to Supun Jayasinghe; hjayasin@ryerson.ca
}

Received 1 October 2017; Revised 8 November 2017; Accepted 13 November 2017; Published 15 January 2018

Academic Editor: Nerio Tullini

Copyright (C) 2018 Supun Jayasinghe and Seyed M. Hashemi. This is an open access article distributed under the Creative Commons Attribution License, which permits unrestricted use, distribution, and reproduction in any medium, provided the original work is properly cited.

\begin{abstract}
The Dynamic Finite Element (DFE) formulation is a superconvergent, semianalytical method used to perform vibration analysis of structural components during the early stages of design. It was presented as an alternative to analytical and numerical methods that exhibit various drawbacks, which limit their applicability during the preliminary design stages. The DFE method, originally developed by the second author, has been exploited heavily to study the modal behaviour of beams in the past. Results from these studies have shown that the DFE method is capable of arriving at highly accurate results with a coarse mesh, thus, making it an ideal choice for preliminary stage modal analysis and design of structural components. However, the DFE method has not yet been extended to study the vibration behaviour of plates. Thus, the aim of this study is to develop a set of frequencydependent, trigonometric shape functions for a 4-noded, 4-DOF per node element as a basis for developing a DFE method for thin rectangular plates. To this end, the authors exploit a distinct quasi-exact solution to the plate governing equation and this solution is then used to derive the new, trigonometric basis and shape functions, based on which the DFE method would be developed.
\end{abstract}

\section{Introduction}

For decades, thin plates have been used to model the vibration behaviour of low curvature two-dimensional structures having very small thicknesses compared to the other dimensions. Their versatility has led to them being used especially in the aerospace industry, where they have found extensive applications. At the onset of airframe design, engineers are required to simulate the vibration behaviour of the airframe component to determine the operational range of frequencies and the mode shapes that arise under real-life conditions. When such a modal analysis is carried out, the effects of loading and boundary conditions and the contributions from any nearby vibrating entities are also incorporated as these factors could modify the vibration characteristics of the entire system. Thus, in order to avoid the dangers of resonance that could occur if the operational and resonant frequencies overlap, it is imperative that the results obtained from the preliminary modal analysis are highly accurate.

Among the many methods available for vibration analysis, the analytical methods yield the highest accuracy but one major hurdle in using these methods is that they require the closed form solution to the governing partial differential equation. This can be a very tedious process, if at all a tractable one. To circumvent this problem, many simplifying assumptions have been incorporated in to the existing exact methods and, as a result, they exhibit many limitations. Having lost their generality, these exact methods are then only applicable to specific plate shapes and plates subjected to certain boundary conditions, as briefly discussed below.

For example, the Navier method [1], which is one of the most popular analytical methods, transforms the governing partial differential equation into an algebraic expression by 
using a double Fourier trigonometric series; however, it is only applicable to plates having at least two edges simply supported. Levy $[2,3]$ made a noteworthy contribution to plate vibration analysis by utilizing a single Fourier trigonometric series to solve the governing equation. Nevertheless, the Levy method is also only applicable to plates that have at least two edges simply supported. Furthermore, this method is not applicable to nonrectangular plates and bending-twisting coupling effects could not be investigated. As evident, the implementation of Fourier series expansions to analyse the vibration of plates has been very common due to their orthogonality, completeness, and stability [4]. However, the conventional Fourier series method consists of a convergence problem along the boundary edges due to discontinuities in displacement and its derivatives [4]. Thus, it is a method that is only applicable to a few very simple boundary conditions. In the Improved Fourier Series Method (IFSM), modifications have been made to eliminate all of the discontinuities and accelerate convergence [5]. It has also made this method applicable to many plate types and boundary conditions; however, even with the modifications IFSM still remains an analytical method that falls short in adaptability when applied to study the vibration behaviour of complex, real-life structural configurations that could be approximated as thin plates or thin plate assemblies.

The Dynamic Stiffness Method (DSM) is also another exact method that has been heavily exploited by researchers to study the vibration of a variety of plate configurations. Boscolo and Banerjee [6] used DSM to determine the vibration of plates using both Classical Plate Theory and first-order shear deformation theory. Later, they exploited the DSM method to perform exact in-plane free vibration analysis of plates and plate assemblies [7]. This was followed by the development of an exact spectral-dynamic stiffness method for free flexural vibration analysis of orthotropic composite plate assemblies by Liu and Banerjee $[8,9]$. Using the novel spectral-dynamic stiffness method, the same authors [10] subsequently conducted an investigation into the free vibration of plates subjected to arbitrary boundary conditions. Despite these advancements, the applicability of DSM method remains limited to special cases and plates subjected to simple boundary conditions. It also requires extensive equation reformulation to accommodate any changes made to the structural configuration.

Numerical methods such as the conventional finite element method (FEM) are heavily used for modal analysis. However, FEM requires a large number of elements to converge to an accurate solution, especially for higher modes as they are based on polynomial shape functions [11]. Despite the need for a large number of elements, the comprehensive nature of conventional FEM makes it more suitable for use during the advanced stages of design where the structural complexity increases and a more detailed analysis of the vibrational characteristics of the structure is required. However, during the early stages of design, where minimizing time and resources consumed on preliminary analysis is as important an objective as the accuracy of the results itself, conventional FEM fails to meet its objective as it cannot produce results to an acceptable degree of precision with a small number of elements. For higher mode numbers and for larger structures this error increases exponentially. Thus, this inconsistency in accuracy and rate of convergence calls for a new method that is tailored for the demands of the early stages of design. That is, a method that yields results both swiftly and to an highly accurate ballpark.

Another frequently used collocation scheme is the Differential Quadrature (DQ) method first introduced by Bellman et al. $[12,13]$ in the early 1970 s. It is not only simple, but also easy to implement and use [14]. Also, it is capable of yielding highly accurate results for lower frequencies using a considerably smaller number of grid points thus consuming less virtual storage and computational effort [14]. Despite these advantages, one of the major drawbacks of this method is that it is difficult to apply to problems involving differential equations with multiple end conditions at the system (line) boundaries such as the fourth-order differential equation for thin plates [15]. Another disadvantage of the DQ method is that its accuracy drops when applied to study the vibration behaviour of complicated geometries [16].

It is here that the importance of the Dynamic Finite Element (DFE) method, as an alternative semianalytical procedure for vibration analysis and modelling of structural components during the preliminary design stages, is realized. Although relatively new, the DFE method is a wellestablished, semianalytical method developed by Hashemi [17]. Since its inception, the DFE method has been used in beam, beam-like, and blade vibration modelling and analysis. Hashemi and his coworkers (see, e.g., [18-20]) have extensively studied the free vibration of various beam configurations, such as isotropic, sandwich, composite, and thin-walled beams subjected to diverse loading configurations, using the Dynamic Finite Element (DFE) method. The results have consistently shown the DFE method to have a higher accuracy and rate of convergence compared to conventional FEM owing to the increased efficiency of the frequency-dependent, trigonometric shape functions based on the exact solutions to the governing equation which the DFE method employs. In one study [19], application of the DFE formulation to the free vibration analysis of a sandwich beam resulted in a quasi-exact formulation. In a more recent study [20], investigating the vibration of bending-torsion coupled beams subjected to axial load and end moment, it was found that the DFE method required eight times less elements than conventional FEM to produce results with a percent difference of less than one percent. However, the DFE method has not yet been fully extended to model plate vibration problems. As the above-mentioned contrasting choice of shape functions is the main distinction between the DFE and FEM methods, the logical first step towards developing a DFE solution to the thin plate problem is to formulate the pertinent dynamic shape functions. With that in mind, the primary objective of this study is to develop a set of viable and robust frequency-dependent, trigonometric shape functions for a 4-noded, 4-DOF per node element and to this end a quasi-exact solution to the thin rectangular plate governing equation was pursued by following a unique approach 


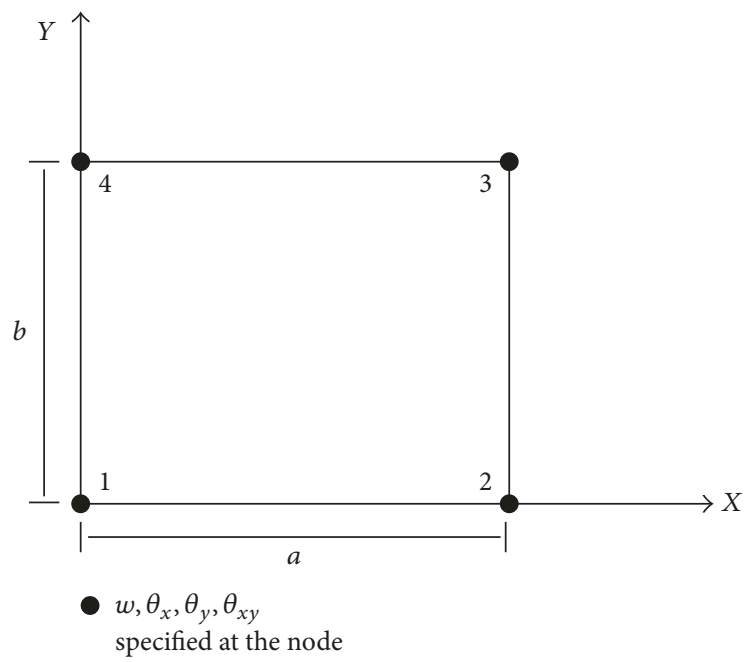

FIgURE 1: Conforming four-node rectangular elements with $16-$ DOF ensuring $C^{1}$ continuity.

that, to the best of the authors' knowledge, has never been presented.

\section{Theoretical Background}

Consider the 4-noded, 4-DOF per node rectangular element shown in Figure 1. The four degrees of freedom are flexural displacement $w$, slope in the $x$-direction, $\theta_{x}$, slope in the $y$ direction, $\theta_{y}$, and curvature, $\theta_{x y}$. It is an element that gives $C^{1}$ continuity, in that, along the element interfaces, there is continuity of $w$ as well as the first derivative of $w$ normal to the interface $(\partial w / \partial n)$. Having four nodes with 4 -DOF per node requires 16 shape functions; however, in order to form the shape dynamic shape functions it is important to find the quasi-exact solutions for the plate governing equation. In an earlier conference publication [21], the authors presented the first four trigonometric shape functions determined during the preliminary stages of this research, along with a very brief introduction of the quasi-exact solution process. However, for further clarity the details of the quasi-exact solution are explicitly outlined below. Thus, from Classical Plate Theory (CPT) the governing general equation for a thin rectangular plate could be written as shown [22] in

$$
D\left(\frac{\partial^{4} w}{\partial x^{4}}+2 \frac{\partial^{4} w}{\partial x^{2} \partial y^{2}}+\frac{\partial^{4} w}{\partial y^{4}}\right)+\rho h \frac{\partial^{2} w}{\partial t^{2}}=0,
$$

where $\rho$ is the mass density, $h$ is the thickness of the plate, and $D$ represents the plate modulus defined as

$$
D=\frac{E h^{3}}{12\left(1-v^{2}\right)} \text {. }
$$

In (2), $E$ is Young's Modulus of the plate and $v$ is Poisson's ratio. form:

If the transformation shown in (3) takes the following

$$
w(x, y, t)=W(x, y) e^{i \omega t} ; \quad \text { where } W(x, y)=e^{A x} e^{B y}
$$

then the characteristic equation for the governing differential can be written as

$$
A^{4}+2 A^{2} B^{2}+B^{4}-\frac{\omega^{2} \rho h}{D}=0 .
$$

Decomposing the plate equation into two separate beam-like expressions representing each spatial coordinate direction of the plate gives the following expression.

$$
\underbrace{\left(A^{4}+A^{2} B^{2}-k_{1} \frac{\omega^{2} \rho h}{D}\right)}_{* 0 .}+\underbrace{\left(B^{4}+A^{2} B^{2}-k_{2} \frac{\omega^{2} \rho h}{D}\right)}_{* *}
$$

In (5), $k_{1}$ and $k_{2}$ are the mass distribution constants along the $x$ - and $y$-directions, respectively. The ratio $k_{1} / k_{2}$ could be described as the aspect ratio of the plate. As shown in (5), by introducing these constants the governing partial differential equation could be decomposed and rewritten as the sum of two beam-like expressions $(*)$ and $(* *)$. Expressing the plate governing equation as a sum of the two beam-like expressions shown in (5) makes the process of determining the roots much simpler. Two constraints were stipulated at the outset of introducing the mass distribution constants in order to maintain the integrity of the plate governing equation and those are as follows. The constants $k_{1}$ and $k_{2}$ could take any positive numerical value between 0 and 1 . However, the sum of the two constants should be equal to one (i.e., $0<k_{1}$ and $k_{2}<1$, and $\left.k_{1}+k_{2}=1\right)$.

It is important to note here that the values taken by the mass distribution constants will dictate the shape of the plate. For example, both $k_{1}$ and $k_{2}$ being equal to 0.5 would represent a square plate and other values of $k_{1}$ and $k_{2}$ would yield various rectangular plate shapes. In both expressions $(*)$ and $(* *)$ of (5), $A$ is considered to be the spatial variable in the $x$-direction. Similarly, $B$ is treated as the spatial variable in the $y$-direction. Each expression $(*)$ and $(* *)$ in $(5)$ is treated as an individual equation and the quadratic formula is applied on each one of them separately to determine the roots. When applying the quadratic formula on expression (*), $A$ is allowed to vary and $B$ is held constant. Similarly, when determining the solutions for expression $(* *), B$ is allowed to vary and $A$ is treated as a constant. After mathematical manipulation and simplification the following roots are found for expression $(*)$ of the plate governing 
equation.

$$
A=\sqrt{ \pm k_{1} \omega \sqrt{\frac{\rho h}{D}}}
$$

Similarly, the roots for the term $(* *)$ in $(5)$ would be

$$
B=\sqrt{ \pm k_{2} \omega \sqrt{\frac{\rho h}{D}}}
$$

The mathematical manipulations involved in arriving at the roots shown in (6) and (7) are not included here for brevity. Thus, (6) will give four roots, $A_{i}(i=1,2,3,4)$, for the expression $(*)$. These roots, of which two are real and two are imaginary, are defined in

$$
\begin{aligned}
& A_{1}=-A_{3}=\sqrt{k_{1} \omega \sqrt{\frac{\rho h}{D}}}=\beta_{x}, \\
& A_{2}=-A_{4}=i \sqrt{k_{1} \omega \sqrt{\frac{\rho h}{D}}}=\alpha_{x} .
\end{aligned}
$$

Similarly, (7) yields four roots, $B_{i}(i=1,2,3,4)$, for the expression $(* *)$. Once again, out of these four roots two are real and two are imaginary and they are defined in

$$
\begin{aligned}
& B_{1}=-B_{3}=\sqrt{k_{2} \omega \sqrt{\frac{\rho h}{D}}}=\beta_{y}, \\
& B_{2}=-B_{4}=i \sqrt{k_{2} \omega \sqrt{\frac{\rho h}{D}}}=\alpha_{y} .
\end{aligned}
$$

It is important to note here that the roots shown in (8), (9), (10), and (11) satisfy their individual expressions separately but together any real-real or imaginary-imaginary combination $\left(A_{i}\right.$ and $\left.B_{i}\right)$ of these roots also satisfies (5) in general. Thus, each real-real and imaginary-imaginary pair of roots $\left(A_{i}\right.$ and $\left.B_{i}\right)$ is an exact solution to the plate governing equation and there are 8 such pairs of exact solutions. However, if a real-imaginary combination of roots $\left(A_{i}\right.$ and $B_{i}$ ) is substituted in to the characteristic equation, it could be seen that such a pair does not satisfy the entire characteristics equation in general, although on their own each of these roots satisfies its respective beam-like expressions $(*)$ and $(* *)$. There are 8 such real-imaginary combinations that could be made from the solutions presented in (8), (9), (10), and (11) and these pairs $\left(A_{i}\right.$ and $\left.B_{i}\right)$ are not exact solution to the plate governing equation. Thus, out of the 16 combinations of roots that could be developed, 8 satisfy the governing equation fully, but the other 8 fail to do so and as such the solution becomes a quasi-exact solution to the plate governing equation.

Since the solution is assumed to take the form defined by (3), the final 16-term quasi-exact solution for a thin plate could be written as follows.

$$
w(x, y)=\sum_{i=1}^{4} \sum_{j=1}^{4} C_{i} e^{A_{i} x} D_{j} e^{B_{j} y}=\sum_{i=1}^{4} \sum_{j=1}^{4} E_{i j} e^{A_{i} x} e^{B_{j} y}
$$

where $E_{i j}$ in (12) are the unknown coefficients defined in

$$
E_{i j}=C_{i} \cdot D_{j}
$$

Thus, the nonnodal approximation of the solution function, $W$, and the test function, $\delta W$, written in terms of generalized parameters are as follows.

$$
\begin{gathered}
W=\langle P(\xi, \eta)\rangle_{f} *\{a\}, \\
\delta W=\langle P(\xi, \eta)\rangle_{f} *\{\delta a\},
\end{gathered}
$$

where $\xi$ and $\eta$ are the nondimensionalized natural coordinates in the $x$ - and $y$-directions, respectively.

The basis functions of the approximation space are shown in Table 1. These basis functions are designed as combinations of the solutions to the characteristic equations as they are derived from the quasi-exact solution of the governing equation. These basis functions have been developed such that when the natural frequency $\omega$ and subsequently roots, $\alpha_{x}, \alpha_{y}, \beta_{x}$, and $\beta_{y}$, of the characteristic equations tend to zero, the resulting basis functions change to those of a standard thin plate element in the classical FEM, incomplete quintic polynomial [11].

The roots $\beta_{x}, \alpha_{x}, \beta_{y}$, and $\alpha_{y}$ were defined previously and marked as expressions (8) through (11), respectively. The expansion terms in Table 1 could be more concisely written as follows.

$$
\langle P(\omega)\rangle_{f}=\left\langle\begin{array}{llllllllllllllll}
b_{1} & b_{2} & b_{3} & b_{4} & b_{5} & b_{6} & b_{7} & b_{8} & b_{9} & b_{10} & b_{11} & b_{12} & b_{13} & b_{14} & b_{15} & b_{16}
\end{array}\right\rangle .
$$

Replacing the generalized parameters, $\langle a\rangle$ and $\langle\delta a\rangle$ in (14), with the nodal variables, $\left\langle w_{1} \theta_{x 1} \theta_{y 1} \theta_{x y 1}, \ldots, w_{4} \theta_{x 4} \theta_{y 4} \theta_{x y 4}\right\rangle$ and $\left\langle\delta w_{1} \delta \theta_{x 1} \delta \theta_{y 1} \delta \theta_{x y 1}, \ldots, \delta w_{4} \delta \theta_{x 4} \delta \theta_{y 4} \delta \theta_{x y 4}\right\rangle$, and rewriting (14) will result in

$$
\begin{gathered}
\left\{W_{n}\right\}=\left[P_{n}\right]_{f}\{a\}, \\
\left\{\delta W_{n}\right\}=\left[P_{n}\right]_{f}\{\delta a\} .
\end{gathered}
$$

The matrix $\left[P_{n}\right]_{f}$ is defined as 
TABLE 1: Trigonometric basis functions for thin plate.

\begin{tabular}{|c|c|c|}
\hline Symbol & FEM & DFE \\
\hline$b_{1}$ & 1 & $\cos \left(\alpha_{x} \xi\right) \cos \left(\alpha_{y} \eta\right)$ \\
\hline \multirow{2}{*}{$b_{2}$} & \multirow{2}{*}{$x$} & $\left(\cos \left(\alpha_{y} \eta\right) \sin \left(\alpha_{x} \xi\right)\right)$ \\
\hline & & $\begin{array}{c}\alpha_{x} \\
\left(\cos \left(\alpha_{x} \xi\right) \sin \left(\alpha_{y} \eta\right)\right)\end{array}$ \\
\hline$b_{3}$ & $y$ & $\cosh \left(\beta_{x} \xi\right) \cos \left(\alpha_{y} \eta\right)-\cos \left(\alpha_{x} \xi\right) \cos \left(\alpha_{y} \eta\right)$ \\
\hline$b_{4}$ & $x^{2}$ & $\begin{array}{c}\alpha_{x}^{2}+\beta_{x}^{2} \\
\left(\sin \left(\alpha_{x} \xi\right) \sin \left(\alpha_{y} \eta\right)\right)\end{array}$ \\
\hline$b_{5}$ & $x y$ & $\begin{array}{c}\alpha_{x} \alpha_{y} \\
\cosh \left(\beta_{y} \eta\right) \cos \left(\alpha_{x} \xi\right)-\cos \left(\alpha_{y} \eta\right) \cos \left(\alpha_{x} \xi\right)\end{array}$ \\
\hline$b_{6}$ & $y^{2}$ & $\begin{array}{c}\alpha_{y}{ }^{2}+\beta_{y}{ }^{2} \\
\sinh \left(\beta_{x} \xi\right) \cos \left(\alpha_{y} \eta\right)-\sin \left(\alpha_{x} \xi\right) \cos \left(\alpha_{y} \eta\right) \\
\end{array}$ \\
\hline$b_{7}$ & $x^{3}$ & $\begin{array}{c}\alpha_{x}{ }^{3}+\beta_{x}^{3} \\
\cosh \left(\beta_{x} \xi\right) \sin \left(\alpha_{y} \eta\right)-\cos \left(\alpha_{x} \xi\right) \sin \left(\alpha_{y} \eta\right) \\
\end{array}$ \\
\hline$b_{8}$ & $x^{2} y$ & $\begin{array}{c}\alpha_{y} \alpha_{x}^{2}+\alpha_{y} \beta_{x}^{2} \\
\cosh \left(\beta_{y} \eta\right) \sin \left(\alpha_{x} \xi\right)-\cos \left(\alpha_{y} \eta\right) \sin \left(\alpha_{x} \xi\right)\end{array}$ \\
\hline$b_{9}$ & $x y^{2}$ & $\begin{array}{c}\alpha_{x} \alpha_{y}^{2}+\alpha_{x} \beta_{y}^{2} \\
\sinh \left(\beta_{y} \eta\right) \cos \left(\alpha_{x} \xi\right)-\sin \left(\alpha_{y} \eta\right) \cos \left(\alpha_{x} \xi\right)\end{array}$ \\
\hline$b_{10}$ & $y^{3}$ & $\alpha_{y}{ }^{3}+\beta_{y}{ }^{3}$ \\
\hline$b_{11}$ & \multirow[t]{2}{*}{$x^{3} y$} & $\underline{\sinh \left(\beta_{x} \xi\right) \sin \left(\alpha_{y} \eta\right)-\sin \left(\alpha_{x} \xi\right) \sin \left(\alpha_{y} \eta\right)}$ \\
\hline \multirow{2}{*}{$b_{12}$} & & $\begin{array}{c}\alpha_{y} \alpha_{x}{ }^{3}+\alpha_{y} \beta_{x}{ }^{3} \\
\cosh \left(\beta_{x} \xi\right) \cosh \left(\beta_{y} \eta\right)-\cosh \left(\beta_{x} \xi\right) \cos \left(\alpha_{y} \eta\right)-\cos \left(\alpha_{x} \xi\right) \cosh \left(\beta_{y} \eta\right)+\cos \left(\alpha_{x} \xi\right) \cos \left(\alpha_{y} \eta\right)\end{array}$ \\
\hline & $x^{2} y^{2}$ & $\begin{array}{c}\alpha_{x}{ }^{2} \alpha_{y}{ }^{2}+\alpha_{x}{ }^{2} \beta_{y}{ }^{2}+\beta_{x}{ }^{2} \alpha_{y}{ }^{2}+\beta_{x}{ }^{2} \beta_{y}{ }^{2} \\
\sinh \left(\beta_{y} \eta\right) \sin \left(\alpha_{x} \xi\right)-\sin \left(\alpha_{y} \eta\right) \sin \left(\alpha_{x} \xi\right)\end{array}$ \\
\hline$b_{13}$ & $x y^{3}$ & $\alpha_{x} \alpha_{y}^{3}+\alpha_{x} \beta_{y}^{3}$ \\
\hline \multirow{2}{*}{$b_{14}$} & \multirow{2}{*}{$x^{3} y^{2}$} & $\sinh \left(\beta_{x} \xi\right) \cosh \left(\beta_{y} \eta\right)-\sinh \left(\beta_{x} \xi\right) \cos \left(\alpha_{y} \eta\right)-\sin \left(\alpha_{x} \xi\right) \cosh \left(\beta_{y} \eta\right)+\sin \left(\alpha_{x} \xi\right) \cos \left(\alpha_{y} \eta\right)$ \\
\hline & & $\alpha_{x}{ }^{3} \alpha_{y}{ }^{2}+\alpha_{x}{ }^{3} \beta_{y}{ }^{2}+\beta_{x}{ }^{3} \alpha_{y}{ }^{2}+\beta_{x}^{3} \beta_{y}{ }^{2}$ \\
\hline \multirow{2}{*}{$b_{15}$} & \multirow{2}{*}{$x^{2} y^{3}$} & $\cosh \left(\beta_{x} \xi\right) \sinh \left(\beta_{y} \eta\right)-\cosh \left(\beta_{x} \xi\right) \sin \left(\alpha_{y} \eta\right)-\cos \left(\alpha_{x} \xi\right) \sinh \left(\beta_{y} \eta\right)+\cos \left(\alpha_{x} \xi\right) \sin \left(\alpha_{y} \eta\right)$ \\
\hline & & $\alpha_{x}{ }^{2} \alpha_{y}{ }^{3}+\alpha_{x}{ }^{2} \beta_{y}{ }^{3}+\beta_{x}{ }^{2} \alpha_{y}{ }^{3}+\beta_{x}{ }^{2} \beta_{y}{ }^{3}$ \\
\hline \multirow{2}{*}{$b_{16}$} & \multirow{2}{*}{$x^{3} y^{3}$} & $\sinh \left(\beta_{x} \xi\right) \sinh \left(\beta_{y} \eta\right)-\sinh \left(\beta_{x} \xi\right) \sin \left(\alpha_{y} \eta\right)-\sin \left(\alpha_{x} \xi\right) \sinh \left(\beta_{y} \eta\right)+\sin \left(\alpha_{x} \xi\right) \sin \left(\alpha_{y} \eta\right)$ \\
\hline & & $\alpha_{x}{ }^{3} \alpha_{y}{ }^{3}+\alpha_{x}{ }^{3} \beta_{y}{ }^{3}+\beta_{x}{ }^{3} \alpha_{y}{ }^{3}+\beta_{x}{ }^{3} \beta_{y}{ }^{3}$ \\
\hline
\end{tabular}

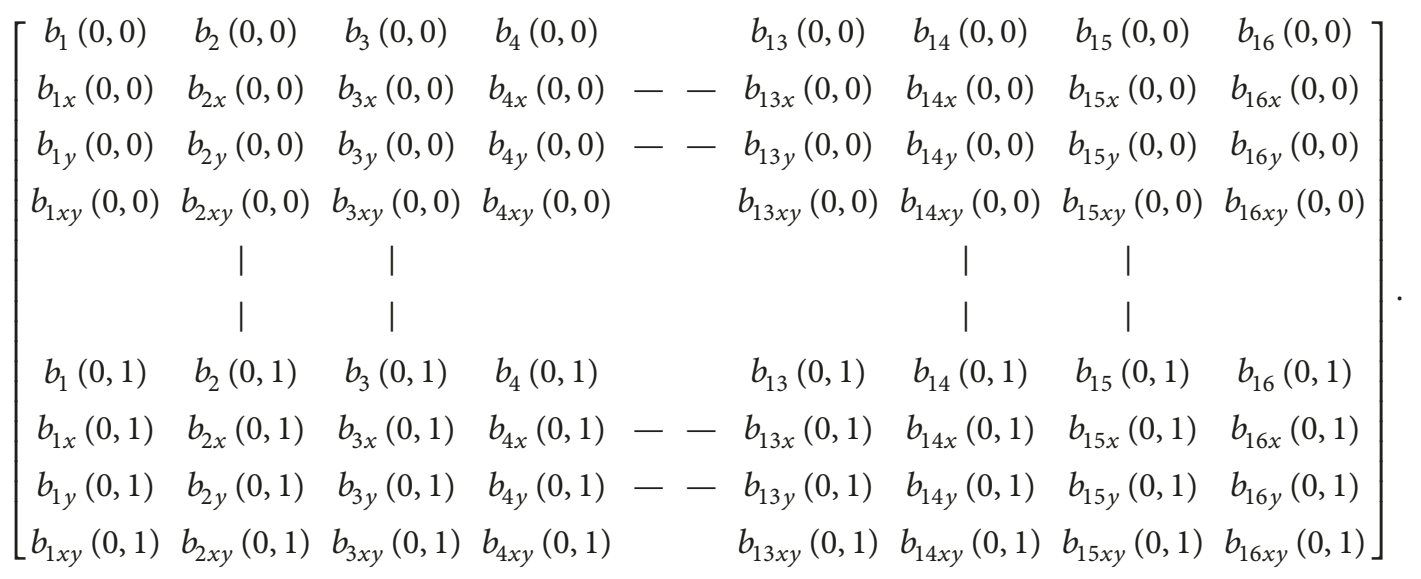




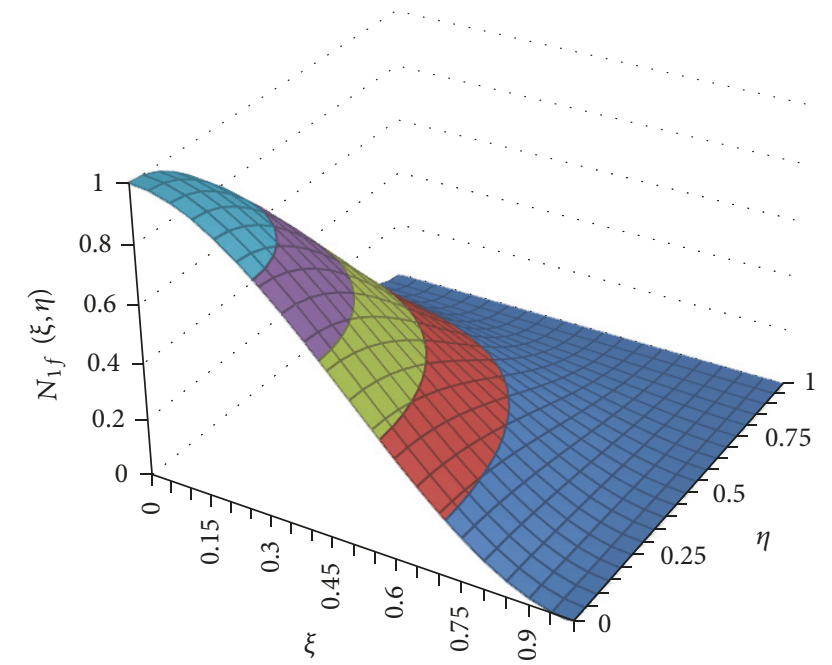

(a)

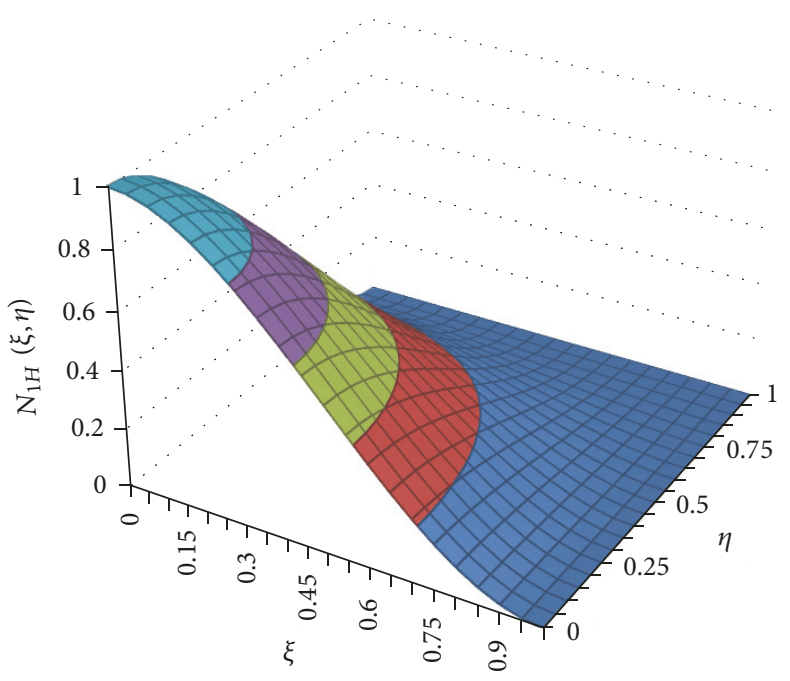

(b)

FIgURE 2: DFE (a) and FEM (b) shape function for $w_{1}$ at node 1.

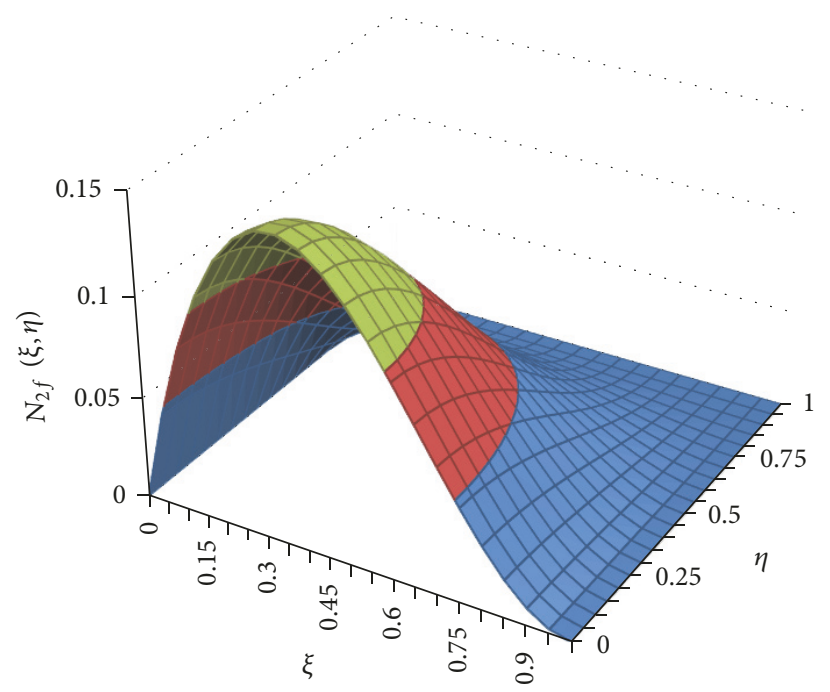

(a)

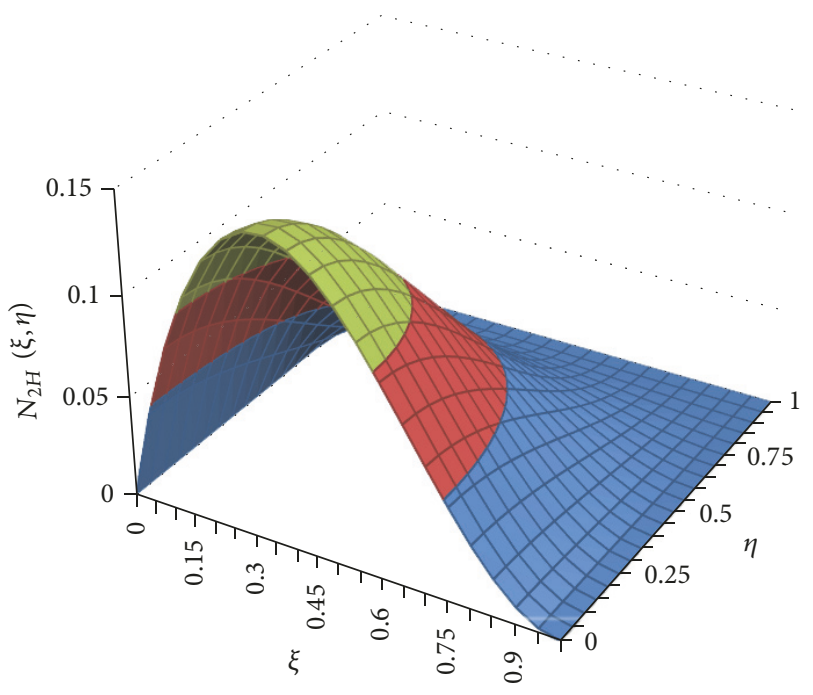

(b)

Figure 3: DFE (a) and FEM (b) shape function for $\theta_{x 1}$ at node 1.

Thus, (14) and the $\left[P_{n}\right]_{f}$ matrix in (17) could be combined in the following manner to construct nodal approximations for flexural displacement, $W(\xi, \eta)$.

$$
\begin{aligned}
W(\xi, \eta) & =\langle P(\xi, \eta)\rangle_{f}\left[P_{n}\right]_{f}^{-1}\left\{w_{n}\right\} \\
& =\langle N(\xi, \eta)\rangle_{f}\left\{w_{n}\right\} .
\end{aligned}
$$

In (18), $\langle N(\xi, \eta)\rangle_{f}$ is the frequency-dependent trigonometric shape function for flexure which could also be rewritten as

$$
\{W(\xi, \eta)\}=\langle N(\xi, \eta)\rangle\left\{w_{n}\right\}
$$

where

$$
\begin{gathered}
\left\{w_{n}\right\}=\left\langle w_{1}, \theta_{1 x}, \theta_{1 y}, \theta_{1 x y}, \ldots, w_{4}, \theta_{4 x}, \theta_{4 y}, \theta_{4 x y}\right\rangle^{T}, \\
\langle N(\xi, \eta)\rangle=\left\langle N_{1 f}, N_{2 f}, N_{3 f}, N_{4 f}, N_{5 f}, N_{6 f}, N_{7 f}, N_{8 f},\right. \\
\left.N_{9 f}, N_{10 f}, N_{11 f}, N_{12 f}, N_{13 f}, N_{14 f}, N_{15 f}, N_{16 f}\right\rangle .
\end{gathered}
$$

The definitions of the frequency-dependent trigonometric shape functions for flexure, $N_{1}$ through $N_{16}$, are explicitly presented in expression (A.1) in Appendix. Also, it is important to emphasize that these new DFE shape functions, which have not been presented for a thin plate element, are unique in their own right and are entirely different from the DFE shape functions for a beam [17-20] presented by 


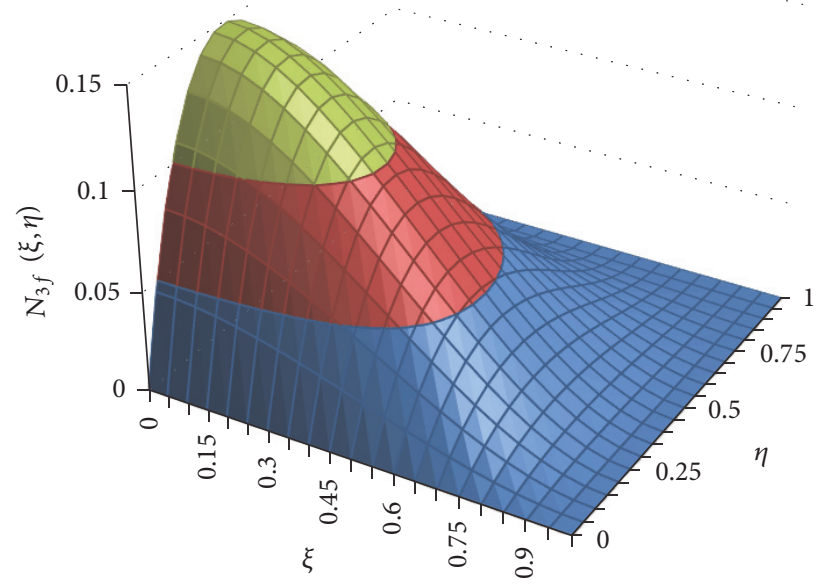

(a)

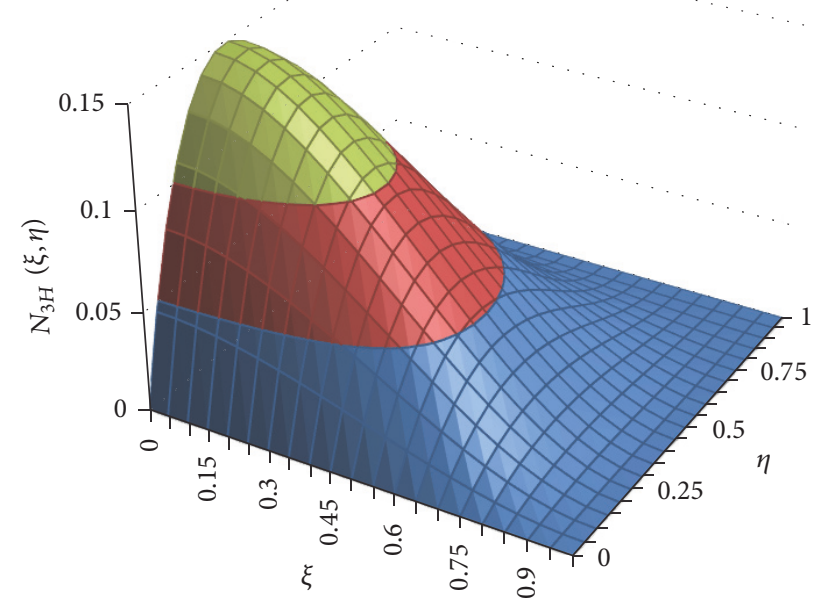

(b)

FIgURE 4: DFE (a) and FEM (b) shape function for $\theta_{y 1}$ at node 1.

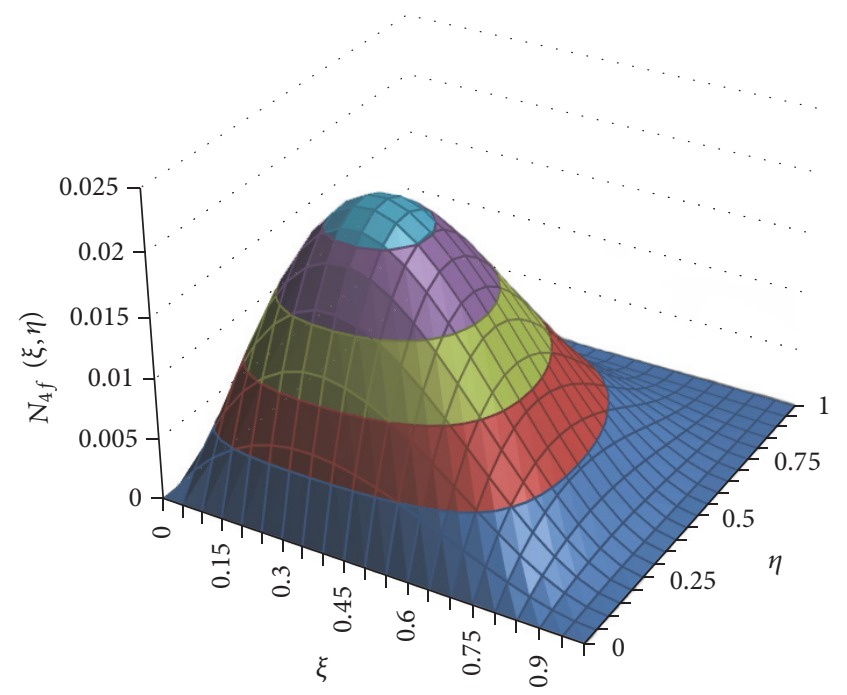

(a)

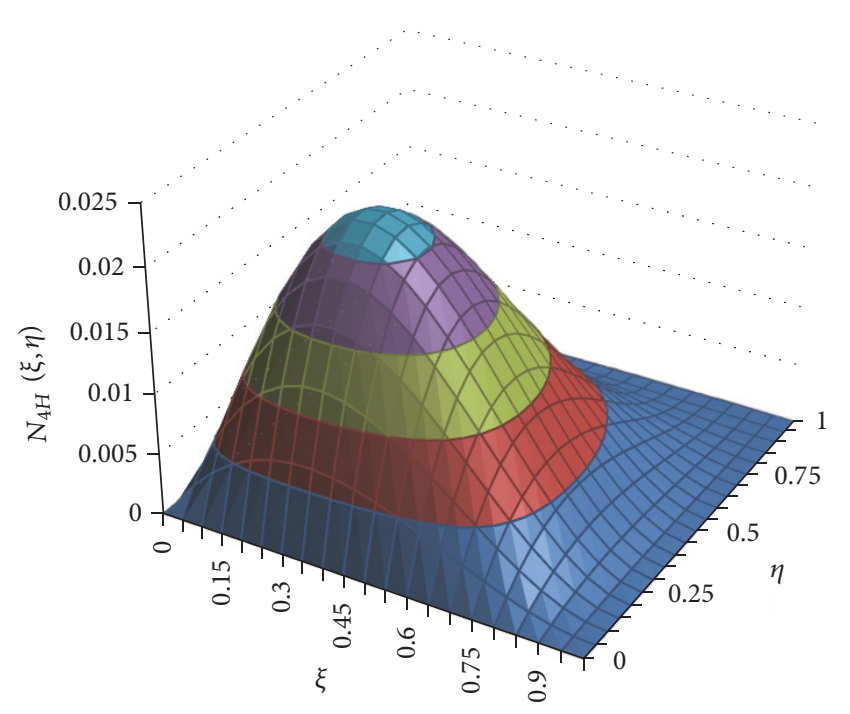

(b)

FIGURE 5: DFE (a) and FEM (b) shape function for $\theta_{x y 1}$ at node 1.

Hashemi and his coworkers in the past. Although developing the QDFE method for a thin rectangular plate, currently in the final stages of its development, is beyond the scope of this paper, for the readers' interest it could be stated that having derived the frequency-dependent, trigonometric shape functions, developing the Quasi-Exact Dynamic Finite Element method now becomes a four-step process, first of which is the application of further integration by parts to the discretized form of the governing differential equation from the conventional FEM formulation. The second step would be to use this further integrated equation together with the DFE shape functions presented here to develop the element stiffness matrices. Once the element matrices are formed, they are assembled using an assembly scheme similar to conventional FEM, and the system boundary conditions are reinforced as the penultimate step to form the global stiffness matrix and the nonlinear Eigenvalue problem. Finally, the frequency domain is swept to determine frequencies that would result in a zero determinant for the system's (global) dynamic stiffness matrix. These are the Eigenfrequencies of the plate and their corresponding Eigenvectors are the mode shapes of the system.

\section{Results and Discussion}

The sixteen new dynamic (frequency-dependent), Trigonometric Flexural Shape Functions (DTFSF) for nodes 1 to 4 of the 16-DOF thin plate element (Figure 1) are plotted next 


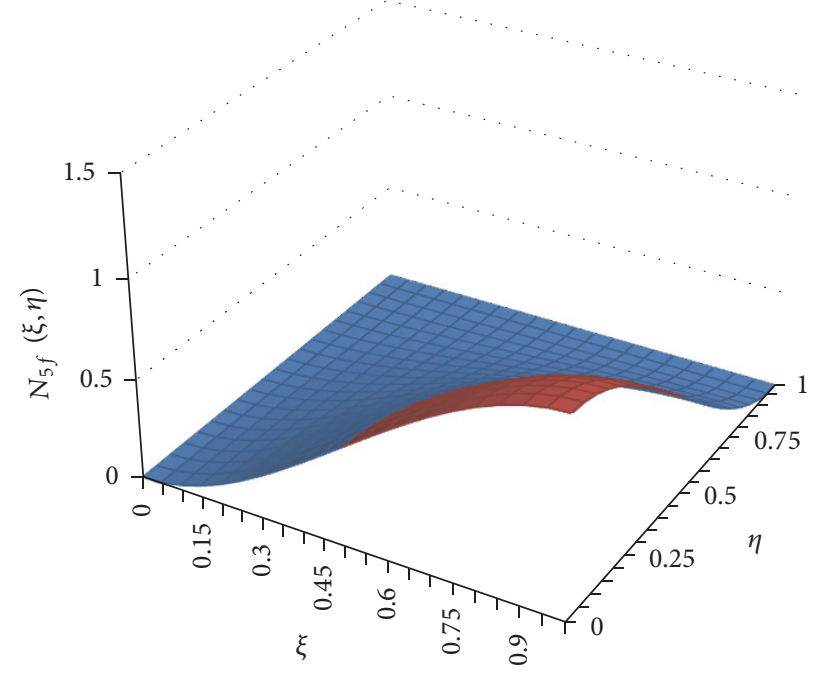

(a)

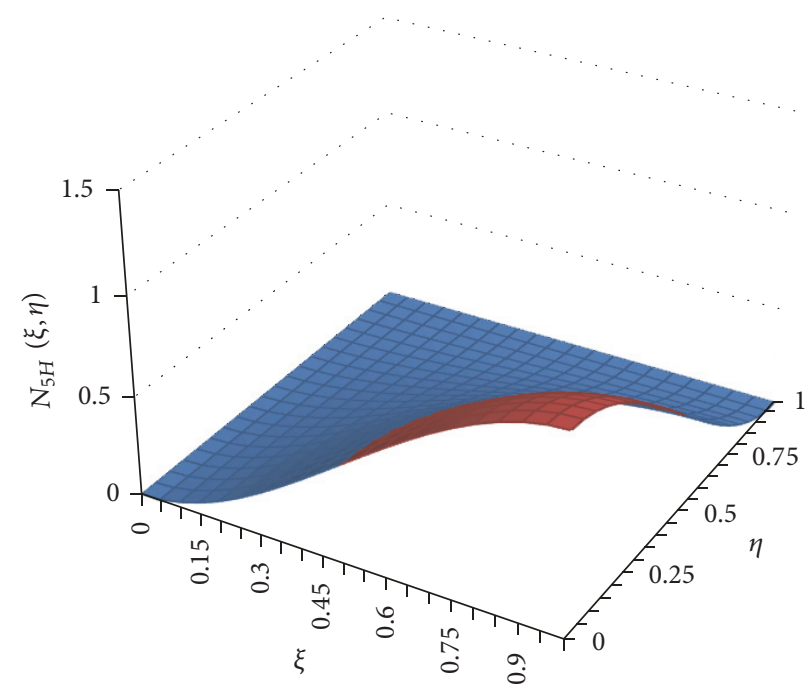

(b)

FIgURE 6: DFE (a) and FEM (b) shape function for $w_{2}$ at node 2.

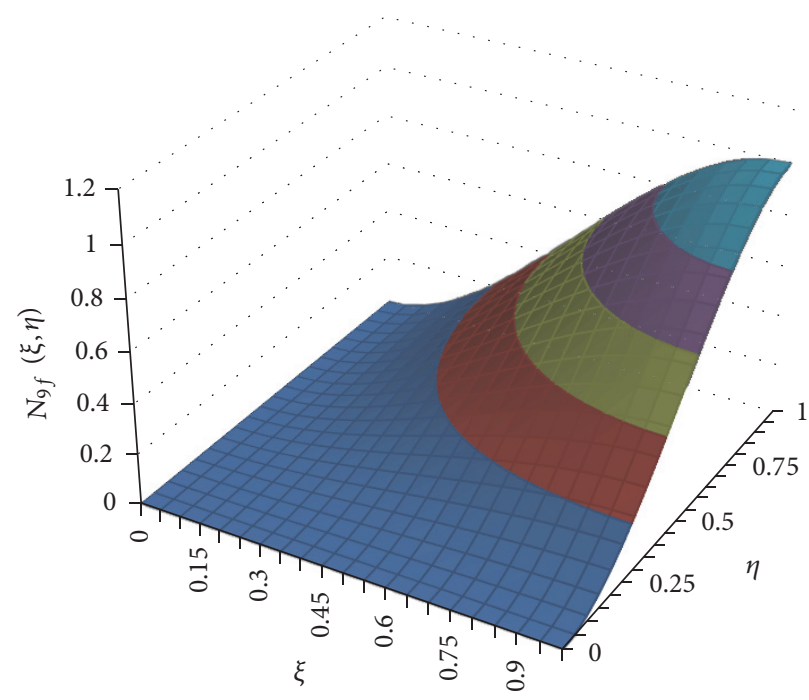

(a)

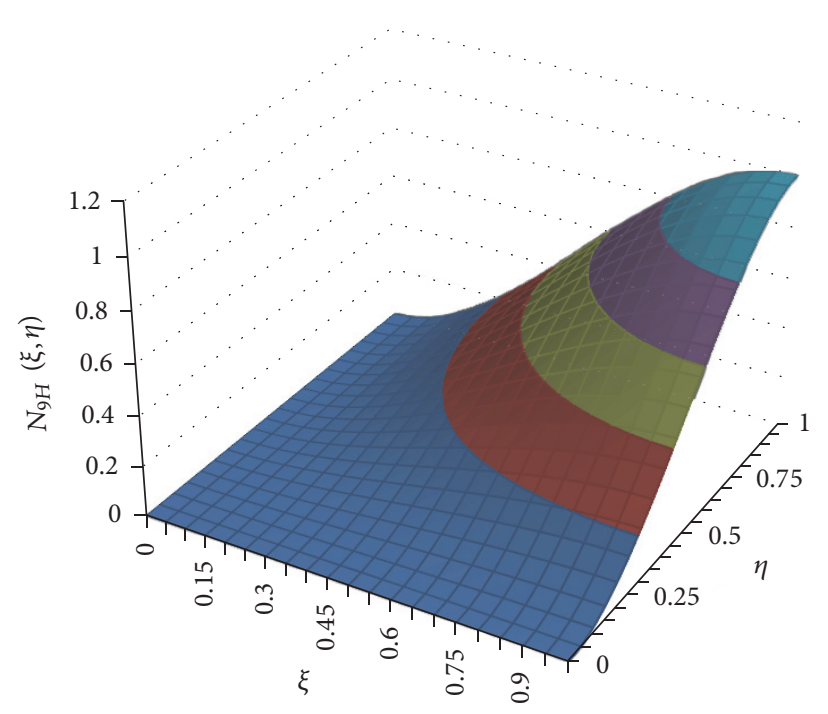

(b)

FIGURE 7: DFE (a) and FEM (b) shape function for $w_{3}$ at node 3 .

to the corresponding FEM (incomplete quantic) polynomial shape functions [11] in Figures 2-8. The DTFSF plots have been created for the following parameters: $E=200 \mathrm{GPa}, \rho$ $=7800 \mathrm{~kg} / \mathrm{m}^{3}, h=0.004 \mathrm{~m}, v=0.3, k_{1}=0.6, k_{2}=0.4$, and a low frequency of $9.36 \mathrm{~Hz}$. As expected, regardless of the frequency, $\omega$, for both the DTFSF and FEM shape functions the flexural displacement $w_{1}$ is 1 at node 1 , where the natural coordinates $\xi$ and $\eta$ are zero. The slope $\theta_{x 1}(\partial w / \partial \xi)$ is also 1 at node 1 for both shape functions $N_{2 f}$ and $N_{2 H}$. Here, the subscript $f$ denotes the frequency-dependent DTFSF and the subscript $H$ stands for the polynomial shape functions used in conventional FEM. Similarly, the gradients of the shape functions $N_{3 f}$ and $N_{3 H}$ which approximate the slope $\theta_{y 1}(\partial w / \partial \eta)$ assume a value of 1 at node 1 too. Furthermore, the slope $\theta_{x y 1}$ which could also be expressed as $\partial^{2} w / \partial \xi \partial \eta$ becomes 1 at node 1 for both shape function types. Thus, it is evident from Figures 2-8 that the new DTFSF shape functions are identical to the conventional FEM polynomial shape functions [11] in how the flexural displacements, slopes, and curvatures are approximated.

The new DTFSF shape functions are also frequencydependent. That is, they oscillate and change with varying frequency $\omega$. In order to illustrate their dependency on frequency, the DTFSF shape functions, $N_{1 f}$ to $N_{16 f}$, are also plotted at two different frequencies $\omega_{1}=82.06 \mathrm{~Hz}$ and $\omega_{2}$ $=206.67 \mathrm{~Hz}$, where $\omega_{1}<\omega_{2}$ (see Figures 9-12). It is also important to note that while dependent on the frequency $\omega$ elsewhere, the trigonometric shape functions for bending are 


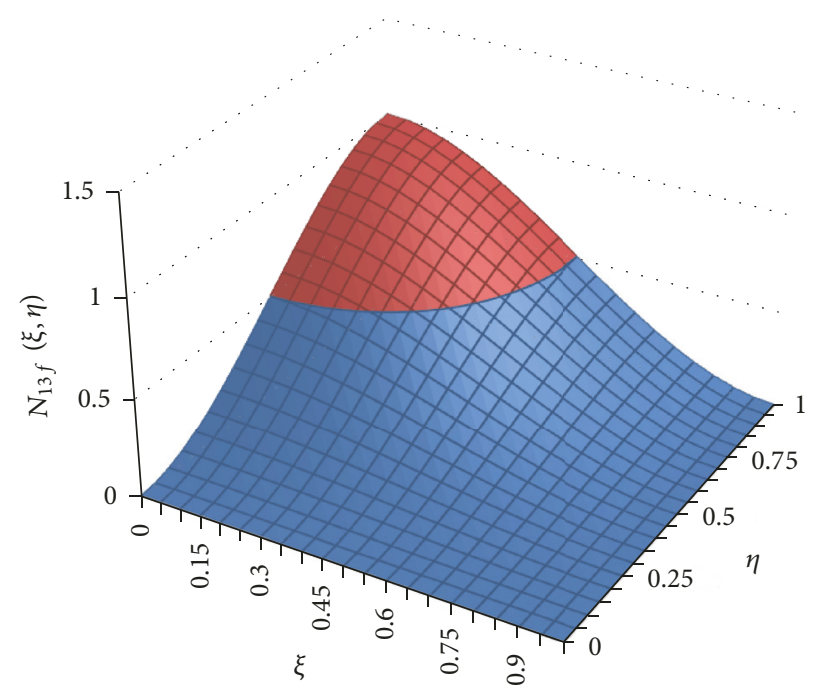

(a)

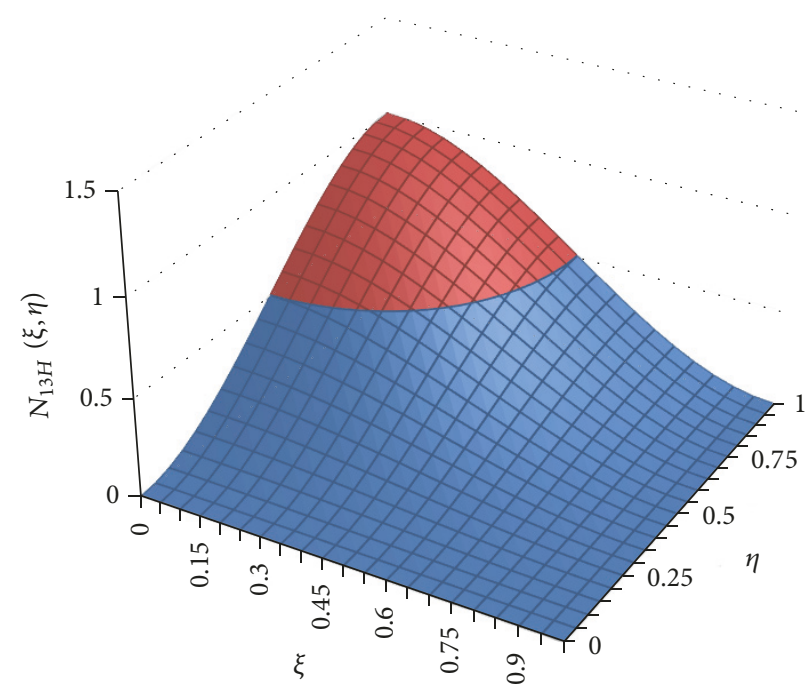

(b)

FIgURE 8: DFE (a) and FEM (b) shape function for $w_{4}$ at node 4.

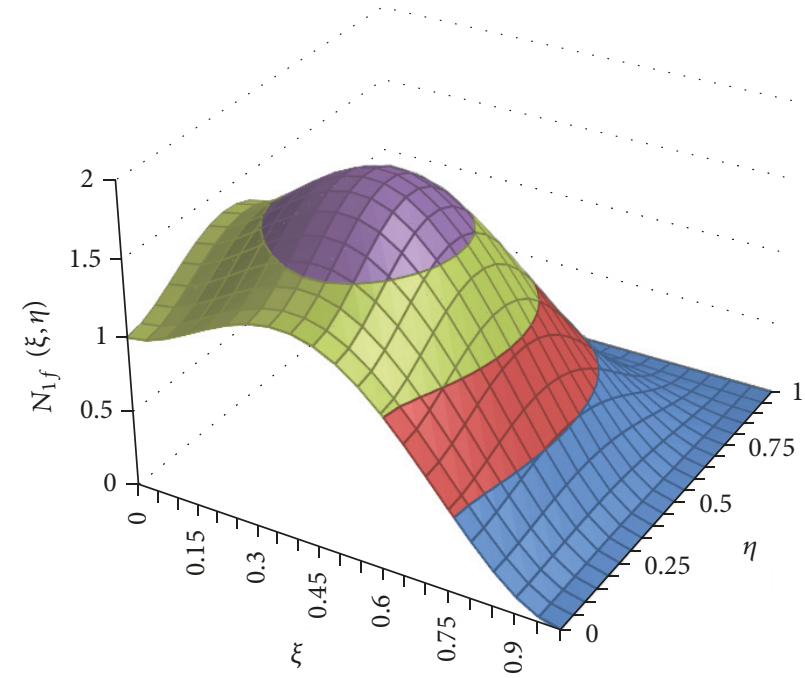

(a)

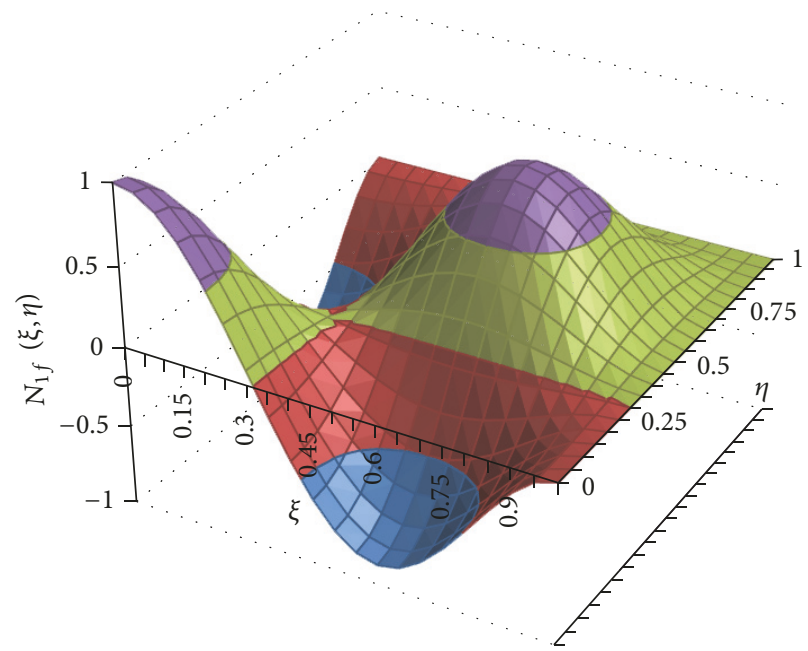

(b)

FIGURE 9: Shape function $N_{1 f}$ at $\omega_{1}$ (a) and $\omega_{2}$ (b).

designed to be independent of the frequency at the element boundaries and as such they take the following values at each node, same as those in the conventional FEM shape functions. At node 1, $N_{1 f}=1$ and $N_{2 f}=N_{3 f}=N_{4 f}=0$. At the second node, $N_{5 f}=1$ and $N_{6 f}=N_{7 f}=N_{8 f}=0$. Similarly at node $3, N_{9 f}=1$ and $N_{10 f}=N_{11 f}=N_{12 f}=0$. Finally, at node $4, N_{13 f}=1$ and $N_{14 f}=N_{15 f}=N_{16 f}=0$.

\section{Conclusion}

In this study a quasi-exact solution was sort for the thin plate governing differential equation based on Classical Plate Theory. The solution was derived by pursuing a unique process whereby the characteristic equations were rearranged as the sum of two beam-like expressions and applying the quadratic formula on each expression separately to determine the roots. Using this quasi-exact solution to the plate governing equation, a set of new trigonometric basis functions were formed such that when the natural frequency tends to zero the trigonometric shape functions tend to their polynomial counterparts used in conventional FEM. Subsequently, sixteen novel dynamic (frequency-dependent) Trigonometric Shape Functions (DTFSF) were developed for a four-node, four-DOF per node rectangular plate element. The identical nature of the DTFSF and polynomial shape functions were then graphically illustrated and the frequencydependent nature of the shape functions was also exemplified. The research is underway to exploit these innovative dynamic bases and shape functions as the framework for a new, QuasiExact Dynamic Finite Element formulation better suited for 


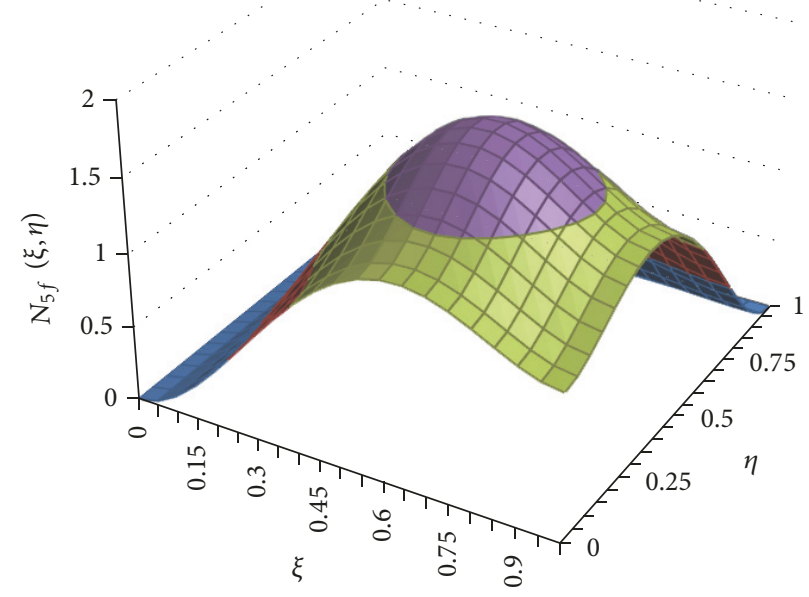

(a)

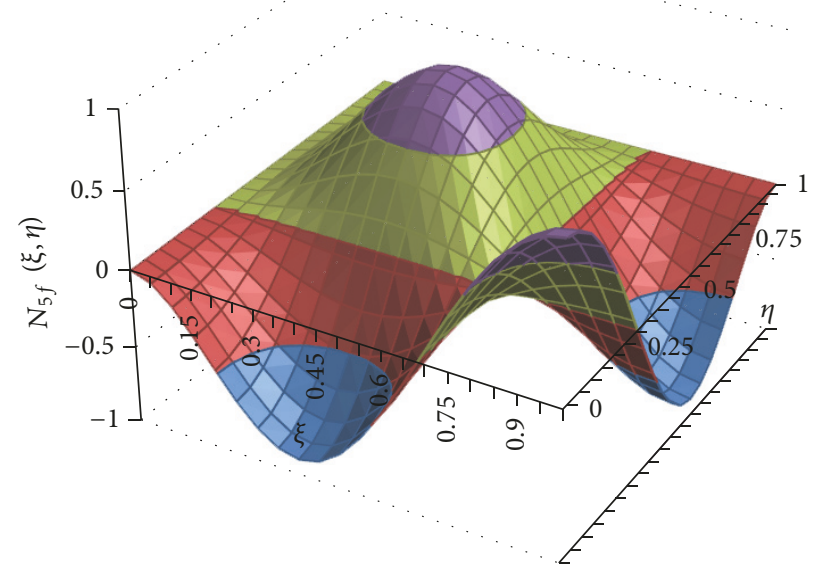

(b)

FIgURE 10: Shape function $N_{5 f}$ at $\omega_{1}$ (a) and $\omega_{2}$ (b).

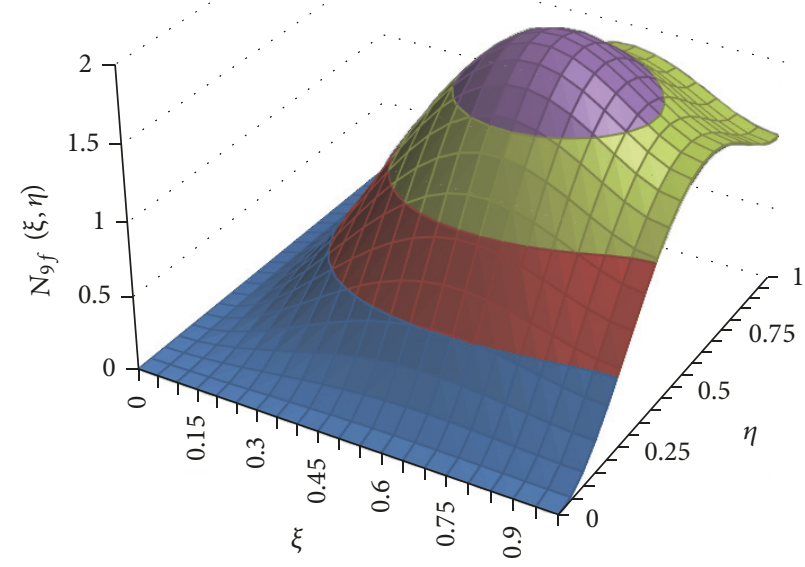

(a)

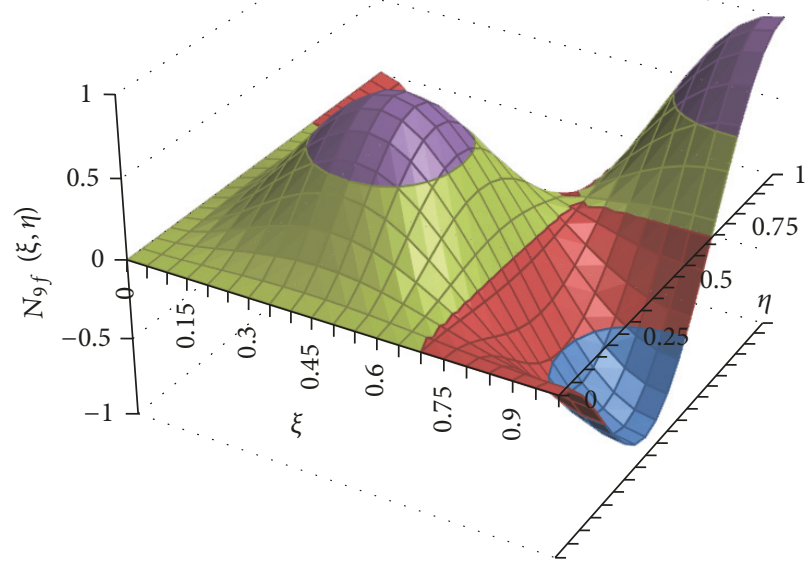

(b)

FIGURE 11: Shape function $N_{9 f}$ at $\omega_{1}$ (a) and $\omega_{2}$ (b).

modelling the vibration behaviour of structural components commonly modelled as thin, rectangular 2D elements during the early stages of design.

\section{Appendix}

\section{Frequency-Dependent Shape Functions}

$$
\begin{gathered}
N_{1 f}=\left\{\left[\cos \left(\alpha_{y} \eta\right)+\cosh \left(\beta_{y} \eta\right)-\cos \left(\alpha_{y}\right)\right.\right. \\
\cdot \cosh \left(\beta_{y}(\eta-1)\right)-\cosh \left(\beta_{y}\right) \cos \left(\alpha_{y}(\eta-1)\right) \\
+\frac{\left(\alpha_{y} \sin \left(\alpha_{y}\right) \sinh \left(\beta_{y}(\eta-1)\right)\right)}{\beta_{y}}
\end{gathered}
$$

$$
\begin{aligned}
& \left.-\frac{\left(\beta_{y} \sinh \left(\beta_{y}\right) \sin \left(\alpha_{y}(\eta-1)\right)\right)}{\alpha_{y}}\right]\left[\cos \left(\alpha_{x} \xi\right)\right. \\
& +\cosh \left(\beta_{x} \xi\right)-\cos \left(\alpha_{x}\right) \cosh \left(\beta_{x}(\xi-1)\right) \\
& -\cosh \left(\beta_{x}\right) \cos \left(\alpha_{x}(\xi-1)\right) \\
& \left.\left.+\frac{\left(\alpha_{x} \sin \left(\alpha_{x}\right) \sinh \left(\beta_{x}(\xi-1)\right)\right)}{\beta_{x}}\right]\right\}\left\{\left[2 \cos \left(\alpha_{x}\right)\right.\right. \\
& \left.-\frac{\left(\beta_{x} \sinh \left(\beta_{x}\right) \sin \left(\alpha_{x}(\xi-1)\right)\right)}{\alpha_{x}}\right) \\
& \cdot \cosh \left(\beta_{x}\right)+\frac{\left(\sin \left(\alpha_{x}\right) \sinh \left(\beta_{x}\right)\left(\alpha_{x}{ }^{2}-\beta_{x}{ }^{2}\right)\right)}{\left(\alpha_{x} \beta_{x}\right)}
\end{aligned}
$$




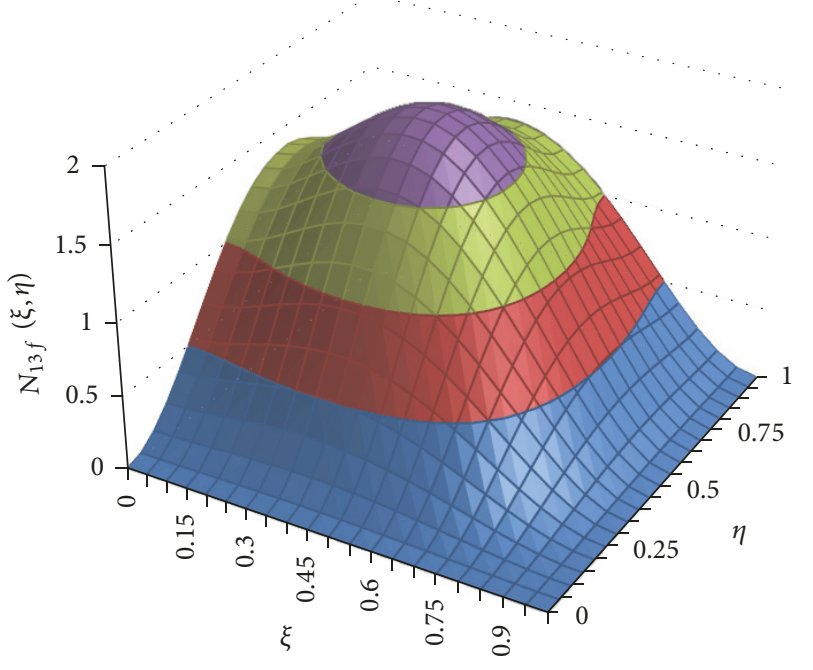

(a)

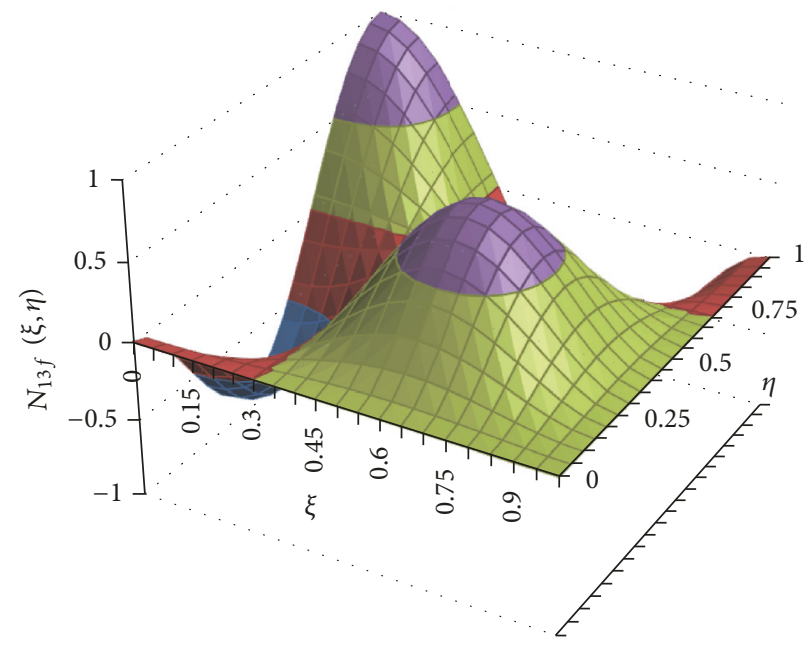

(b)

FIGURE 12: Shape function $N_{13 f}$ at $\omega_{1}(\mathrm{a})$ and $\omega_{2}(\mathrm{~b})$.

$$
\begin{aligned}
&-2]\left[2 \cos \left(\alpha_{y}\right) \cosh \left(\beta_{y}\right)\right. \\
&+\left.\left.\frac{\left(\sin \left(\alpha_{y}\right) \sinh \left(\beta_{y}\right)\left(\alpha_{y}{ }^{2}-\beta_{y}{ }^{2}\right)\right)}{\left(\alpha_{y} \beta_{y}\right)}-2\right]\right\}^{-1}, \\
& N_{2 f}=-\left\{\left[\beta _ { x } \left(\cosh \left(\beta_{x}\right) \sin \left(\alpha_{x}(\xi-1)\right)-\sin \left(\alpha_{x} \xi\right)\right.\right.\right. \\
&\left.+\sin \left(\alpha_{x}\right) \cosh \left(\beta_{x}(\xi-1)\right)\right) \\
&+\alpha_{x}\left(\cos \left(\alpha_{x}\right) \sinh \left(\beta_{x}(\xi-1)\right)-\sinh \left(\beta_{x} \xi\right)\right. \\
&\left.\left.+\sinh \left(\beta_{x}\right) \cos \left(\alpha_{x}(\xi-1)\right)\right)\right] \cos \left(\alpha_{y} \eta\right) \\
&+\cosh \left(\beta_{y} \eta\right)-\cos \left(\alpha_{y}\right) \cosh \left(\beta_{y}(\eta-1)\right) \\
&-\cosh \left(\beta_{y}\right) \cos \left(\alpha_{y}(\eta-1)\right) \\
&+\frac{\left(\alpha_{y} \sin \left(\alpha_{y}\right) \sinh \left(\beta_{y}(\eta-1)\right)\right)}{\beta_{y}} \\
&\left.\left.+\frac{\left(\beta_{y} \sinh \left(\beta_{y}\right) \sin \left(\alpha_{y}(\eta-1)\right)\right)}{\alpha_{y}}\right]\right\}\left\{\alpha_{x} \beta_{x}[2\right.
\end{aligned}
$$$$
\cdot \cos \left(\alpha_{x}\right) \cosh \left(\beta_{x}\right)
$$$$
\left.+\frac{\left(\sin \left(\alpha_{x}\right) \sinh \left(\beta_{x}\right)\left(\alpha_{x}^{2}-\beta_{x}^{2}\right)\right)}{\left(\alpha_{x} \beta_{x}\right)}-2\right][2
$$$$
\cdot \cos \left(\alpha_{y}\right) \cosh \left(\beta_{y}\right)
$$

$$
\left.\left.+\frac{\left(\sin \left(\alpha_{y}\right) \sinh \left(\beta_{y}\right)\left(\alpha_{y}{ }^{2}-\beta_{y}{ }^{2}\right)\right)}{\left(\alpha_{y} \beta_{y}\right)}-2\right]\right\}^{-1},
$$$$
N_{3 f}=-\left\{\left[\beta _ { y } \left(\cosh \left(\beta_{y}\right) \sin \left(\alpha_{y}(\eta-1)\right)\right.\right.\right.
$$$$
\left.-\sin \left(\alpha_{y} \eta\right)+\sin \left(\alpha_{y}\right) \cosh \left(\beta_{y}(\eta-1)\right)\right)
$$$$
+\alpha_{y}\left(\cos \left(\alpha_{y}\right) \sinh \left(\beta_{y}(\eta-1)\right)-\sinh \left(\beta_{y} \eta\right)\right.
$$$$
\left.\left.+\sinh \left(\beta_{y}\right) \cos \left(\alpha_{y}(\eta-1)\right)\right)\right]\left[\cos \left(\alpha_{x} \xi\right)\right.
$$$$
+\cosh \left(\beta_{x} \xi\right)-\cos \left(\alpha_{x}\right) \cosh \left(\beta_{x}(\xi-1)\right)
$$$$
-\cosh \left(\beta_{x}\right) \cos \left(\alpha_{x}(\xi-1)\right)
$$$$
+\frac{\left(\alpha_{x} \sin \left(\alpha_{x}\right) \sinh \left(\beta_{x}(\xi-1)\right)\right)}{\beta_{x}}
$$$$
\left.\left.-\frac{\left(\beta_{x} \sinh \left(\beta_{x}\right) \sin \left(\alpha_{x}(\xi-1)\right)\right)}{\alpha_{x}}\right]\right\}\left\{\alpha_{y} \beta_{y}[2\right.
$$$$
\cdot \cos \left(\alpha_{x}\right) \cosh \left(\beta_{x}\right)
$$$$
\left.+\frac{\left(\sin \left(\alpha_{x}\right) \sinh \left(\beta_{x}\right)\left(\alpha_{x}{ }^{2}-\beta_{x}{ }^{2}\right)\right)}{\left(\alpha_{x} \beta_{x}\right)}-2\right][2
$$

$$
\cdot \cos \left(\alpha_{y}\right) \cosh \left(\beta_{y}\right)
$$$$
\left.\left.+\frac{\left(\sin \left(\alpha_{y}\right) \sinh \left(\beta_{y}\right)\left(\alpha_{y}{ }^{2}-\beta_{y}{ }^{2}\right)\right)}{\left(\alpha_{y} \beta_{y}\right)}-2\right]\right\}^{-1},
$$

$$
N_{4 f}=\left\{\left[\beta _ { y } \left(\cosh \left(\beta_{y}\right) \sin \left(\alpha_{y}(\eta-1)\right)-\sin \left(\alpha_{y} \eta\right)\right.\right.\right.
$$




$$
\begin{aligned}
& \left.+\sin \left(\alpha_{y}\right) \cosh \left(\beta_{y}(\eta-1)\right)\right) \\
& +\alpha_{y}\left(\cos \left(\alpha_{y}\right) \sinh \left(\beta_{y}(\eta-1)\right)-\sinh \left(\beta_{y} \eta\right)\right. \\
& \left.\left.+\sinh \left(\beta_{y}\right) \cos \left(\alpha_{y}(\eta-1)\right)\right)\right] \\
& \text { - }\left[\beta _ { x } \left(\cosh \left(\beta_{x}\right) \sin \left(\alpha_{x}(\xi-1)\right)-\sin \left(\alpha_{x} \xi\right)\right.\right. \\
& \left.+\sin \left(\alpha_{x}\right) \cosh \left(\beta_{x}(\xi-1)\right)\right) \\
& +\alpha_{x}\left(\cos \left(\alpha_{x}\right) \sinh \left(\beta_{x}(\xi-1)\right)-\sinh \left(\beta_{x} \xi\right)\right. \\
& \left.\left.\left.+\sinh \left(\beta_{x}\right) \cdot \cos \left(\alpha_{x}(\xi-1)\right)\right)\right]\right\}\left\{\alpha_{x} \alpha_{y} \beta_{x} \beta_{y}[2\right. \\
& \cdot \cos \left(\alpha_{x}\right) \cosh \left(\beta_{x}\right) \\
& \left.+\frac{\left(\sin \left(\alpha_{x}\right) \sinh \left(\beta_{x}\right)\left(\alpha_{x}{ }^{2}-\beta_{x}{ }^{2}\right)\right)}{\left(\alpha_{x} \beta_{x}\right)}-2\right][2 \\
& \cdot \cos \left(\alpha_{y}\right) \cosh \left(\beta_{y}\right) \\
& \left.\left.+\frac{\left(\sin \left(\alpha_{y}\right) \sinh \left(\beta_{y}\right)\left(\alpha_{y}{ }^{2}-\beta_{y}{ }^{2}\right)\right)}{\left(\alpha_{y} \beta_{y}\right)}-2\right]\right\}^{-1}, \\
& N_{5 f}=\left\{\left[\cos \left(\alpha_{y} \eta\right)+\cosh \left(\beta_{y} \eta\right)-\cos \left(\alpha_{y}\right)\right.\right. \\
& \cdot \cosh \left(\beta_{y}(\eta-1)\right)-\cosh \left(\beta_{y}\right) \cos \left(\alpha_{y}(\eta-1)\right) \\
& +\frac{\left(\alpha_{y} \sin \left(\alpha_{y}\right) \sinh \left(\beta_{y}(\eta-1)\right)\right)}{\beta_{y}} \\
& \left.-\frac{\left(\beta_{y} \sinh \left(\beta_{y}\right) \sin \left(\alpha_{y}(\eta-1)\right)\right)}{\alpha_{y}}\right] \\
& \cdot\left[\cos \left(\alpha_{x}(\xi-1)\right)+\cosh \left(\beta_{x}(\xi-1)\right)\right. \\
& -\cos \left(\alpha_{x} \xi\right) \cosh \left(\beta_{x}\right)-\cosh \left(\beta_{x} \xi\right) \cos \left(\alpha_{x}\right) \\
& -\frac{\left(\alpha_{x} \sinh \left(\beta_{x} \xi\right) \sin \left(\alpha_{x}\right)\right)}{\beta_{x}} \\
& \left.\left.+\frac{\left(\beta_{x} \sin \left(\alpha_{x} \xi\right) \sinh \left(\beta_{x}\right)\right)}{\alpha_{x}}\right]\right\}\left\{\left[2 \cos \left(\alpha_{x}\right)\right.\right. \\
& \cdot \cosh \left(\beta_{x}\right)+\frac{\left(\sin \left(\alpha_{x}\right) \sinh \left(\beta_{x}\right)\left(\alpha_{x}^{2}-\beta_{x}^{2}\right)\right)}{\left(\alpha_{x} \beta_{x}\right)} \\
& -2]\left[2 \cos \left(\alpha_{y}\right) \cosh \left(\beta_{y}\right)\right. \\
& \left.\left.+\frac{\left(\sin \left(\alpha_{y}\right) \sinh \left(\beta_{y}\right)\left(\alpha_{y}{ }^{2}-\beta_{y}{ }^{2}\right)\right)}{\left(\alpha_{y} \beta_{y}\right)}-2\right]\right\}^{-1} \text {, } \\
& \begin{aligned}
N_{6 f} & =\left\{\left[\beta _ { x } \left(\sin \left(\alpha_{x}(\xi-1)\right)+\cosh \left(\beta_{x} \xi\right) \sin \left(\alpha_{x}\right)\right.\right.\right. \\
- & \left.\sin \left(\alpha_{x} \xi\right) \cosh \left(\beta_{x}\right)\right)+\alpha_{x}\left(\sinh \left(\beta_{x}(\xi-1)\right)\right. \\
+ & \left.\left.\cos \left(\alpha_{x} \xi\right) \sinh \left(\beta_{x}\right)-\sinh \left(\beta_{x} \xi\right) \cos \left(\alpha_{x}\right)\right)\right] \\
& \cdot\left[\cos \left(\alpha_{y} \eta\right)+\cosh \left(\beta_{y} \eta\right)-\cos \left(\alpha_{y}\right)\right. \\
\cdot & \cosh \left(\beta_{y}(\eta-1)\right)-\cosh \left(\beta_{y}\right) \cos \left(\alpha_{y}(\eta-1)\right) \\
+ & \left.\left.\frac{\left(\alpha_{y} \sin \left(\alpha_{y}\right) \sinh \left(\beta_{y}(\eta-1)\right)\right)}{\beta_{y}}\right]\right\}\left\{\alpha_{x} \beta_{x}[2\right. \\
& -\frac{\left(\beta_{y} \sinh \left(\beta_{y}\right) \sin \left(\alpha_{y}(\eta-1)\right)\right)}{\alpha_{y}}
\end{aligned} \\
& \cdot \cos \left(\alpha_{x}\right) \cosh \left(\beta_{x}\right) \\
& \left.+\frac{\left(\sin \left(\alpha_{x}\right) \sinh \left(\beta_{x}\right)\left(\alpha_{x}{ }^{2}-\beta_{x}{ }^{2}\right)\right)}{\left(\alpha_{x} \beta_{x}\right)}-2\right][2 \\
& \cdot \cos \left(\alpha_{y}\right) \cosh \left(\beta_{y}\right) \\
& \left.\left.+\frac{\left(\sin \left(\alpha_{y}\right) \sinh \left(\beta_{y}\right)\left(\alpha_{y}{ }^{2}-\beta_{y}{ }^{2}\right)\right)}{\left(\alpha_{y} \beta_{y}\right)}-2\right]\right\}^{-1} \text {, } \\
& N_{7 f}=-\left\{\left[\beta _ { y } \left(\cosh \left(\beta_{y}\right) \sin \left(\alpha_{y}(\eta-1)\right)\right.\right.\right. \\
& \left.-\sin \left(\alpha_{y} \eta\right)+\sin \left(\alpha_{y}\right) \cosh \left(\beta_{y}(\eta-1)\right)\right) \\
& +\alpha_{y}\left(\cos \left(\alpha_{y}\right) \sinh \left(\beta_{y}(\eta-1)\right)-\sinh \left(\beta_{y} \eta\right)\right. \\
& \left.\left.+\sinh \left(\beta_{y}\right) \cos \left(\alpha_{y}(\eta-1)\right)\right)\right]\left[\cos \left(\alpha_{x}(\xi-1)\right)\right. \\
& +\cosh \left(\beta_{x}(\xi-1)\right)-\cos \left(\alpha_{x} \xi\right) \cosh \left(\beta_{x}\right) \\
& -\cosh \left(\beta_{x} \xi\right) \cos \left(\alpha_{x}\right)-\frac{\left(\alpha_{x} \sinh \left(\beta_{x} \xi\right) \sin \left(\alpha_{x}\right)\right)}{\beta_{x}} \\
& \left.\left.+\frac{\left(\beta_{x} \sin \left(\alpha_{x} \xi\right) \sinh \left(\beta_{x}\right)\right)}{\alpha_{x}}\right]\right\}\left\{\alpha _ { y } \beta _ { y } \left[2 \cos \left(\alpha_{x}\right)\right.\right. \\
& \cdot \cosh \left(\beta_{x}\right)+\frac{\left(\sin \left(\alpha_{x}\right) \sinh \left(\beta_{x}\right)\left(\alpha_{x}{ }^{2}-\beta_{x}{ }^{2}\right)\right)}{\left(\alpha_{x} \beta_{x}\right)} \\
& -2]\left[2 \cos \left(\alpha_{y}\right) \cosh \left(\beta_{y}\right)\right. \\
& \left.\left.+\frac{\left(\sin \left(\alpha_{y}\right) \sinh \left(\beta_{y}\right)\left(\alpha_{y}{ }^{2}-\beta_{y}{ }^{2}\right)\right)}{\left(\alpha_{y} \beta_{y}\right)}-2\right]\right\}^{-1} \text {, }
\end{aligned}
$$




$$
\begin{aligned}
& N_{8 f}=-\left\{\left[\beta _ { y } \left(\cosh \left(\beta_{y}\right) \sin \left(\alpha_{y}(\eta-1)\right)\right.\right.\right. \\
& \left.-\sin \left(\alpha_{y} \eta\right)+\sin \left(\alpha_{y}\right) \cosh \left(\beta_{y}(\eta-1)\right)\right) \\
& +\alpha_{y}\left(\cos \left(\alpha_{y}\right) \sinh \left(\beta_{y}(\eta-1)\right)-\sinh \left(\beta_{y} \eta\right)\right. \\
& \left.\left.+\sinh \left(\beta_{y}\right) \cos \left(\alpha_{y}(\eta-1)\right)\right)\right] \\
& \cdot\left[\beta _ { x } \left(\sin \left(\alpha_{x}(\xi-1)\right)+\cosh \left(\beta_{x} \xi\right) \sin \left(\alpha_{x}\right)\right.\right. \\
& \left.-\sin \left(\alpha_{x} \xi\right) \cosh \left(\beta_{x}\right)\right)+\alpha_{x}\left(\sinh \left(\beta_{x}(\xi-1)\right)\right. \\
& \left.\left.\left.+\cos \left(\alpha_{x} \xi\right) \sinh \left(\beta_{x}\right)-\sinh \left(\beta_{x} \xi\right) \cdot \cos \left(\alpha_{x}\right)\right)\right]\right\} \\
& \left\{\alpha _ { x } \alpha _ { y } \beta _ { x } \beta _ { y } \left[2 \cos \left(\alpha_{x}\right) \cosh \left(\beta_{x}\right)\right.\right. \\
& \left.+\frac{\left(\sin \left(\alpha_{x}\right) \sinh \left(\beta_{x}\right)\left(\alpha_{x}{ }^{2}-\beta_{x}^{2}\right)\right)}{\left(\alpha_{x} \beta_{x}\right)}-2\right][2 \\
& \cdot \cos \left(\alpha_{y}\right) \cosh \left(\beta_{y}\right) \\
& \left.\left.+\frac{\left(\sin \left(\alpha_{y}\right) \sinh \left(\beta_{y}\right)\left(\alpha_{y}{ }^{2}-\beta_{y}{ }^{2}\right)\right)}{\left(\alpha_{y} \beta_{y}\right)}-2\right]\right\}^{-1}, \\
& N_{9 f}=\left\{\left[\cos \left(\alpha_{y}(\eta-1)\right)+\cosh \left(\beta_{y}(\eta-1)\right)\right.\right. \\
& -\cos \left(\alpha_{y} \eta\right) \cosh \left(\beta_{y}\right)-\cosh \left(\beta_{y} \eta\right) \cos \left(\alpha_{y}\right) \\
& -\frac{\left(\alpha_{y} \sinh \left(\beta_{y} \eta\right) \sin \left(\alpha_{y}\right)\right)}{\beta_{y}} \\
& \left.+\frac{\left(\beta_{y} \sin \left(\alpha_{y} \eta\right) \sinh \left(\beta_{y}\right)\right)}{\alpha_{y}}\right]\left[\cos \left(\alpha_{x}(\xi-1)\right)\right. \\
& +\cosh \left(\beta_{x}(\xi-1)\right)-\cos \left(\alpha_{x} \xi\right) \cosh \left(\beta_{x}\right) \\
& -\cosh \left(\beta_{x} \xi\right) \cos \left(\alpha_{x}\right)-\frac{\left(\alpha_{x} \sinh \left(\beta_{x} \xi\right) \sin \left(\alpha_{x}\right)\right)}{\beta_{x}} \\
& \left.\left.+\frac{\left(\beta_{x} \sin \left(\alpha_{x} \xi\right) \sinh \left(\beta_{x}\right)\right)}{\alpha_{x}}\right]\right\}\left\{\left[2 \cos \left(\alpha_{x}\right)\right.\right. \\
& \cdot \cosh \left(\beta_{x}\right)+\frac{\left(\sin \left(\alpha_{x}\right) \sinh \left(\beta_{x}\right)\left(\alpha_{x}^{2}-\beta_{x}^{2}\right)\right)}{\left(\alpha_{x} \beta_{x}\right)} \\
& -2]\left[2 \cos \left(\alpha_{y}\right) \cosh \left(\beta_{y}\right)\right. \\
& \left.\left.+\frac{\left(\sin \left(\alpha_{y}\right) \sinh \left(\beta_{y}\right)\left(\alpha_{y}{ }^{2}-\beta_{y}{ }^{2}\right)\right)}{\left(\alpha_{y} \beta_{y}\right)}-2\right]\right\}^{-1} \text {, } \\
& N_{10 f}=\left\{\left[\beta _ { x } \left(\sin \left(\alpha_{x}(\xi-1)\right)+\cosh \left(\beta_{x} \xi\right) \sin \left(\alpha_{x}\right)\right.\right.\right. \\
& \left.-\sin \left(\alpha_{x} \xi\right) \cosh \left(\beta_{x}\right)\right)+\alpha_{x}\left(\sinh \left(\beta_{x}(\xi-1)\right)\right. \\
& \left.\left.+\cos \left(\alpha_{x} \xi\right) \sinh \left(\beta_{x}\right)-\sinh \left(\beta_{x} \xi\right) \cos \left(\alpha_{x}\right)\right)\right] \\
& \cdot\left[\cos \left(\alpha_{y}(\eta-1)\right)+\cosh \left(\beta_{y}(\eta-1)\right)\right. \\
& -\cos \left(\alpha_{y} \eta\right) \cosh \left(\beta_{y}\right)-\cosh \left(\beta_{y} \eta\right) \cos \left(\alpha_{y}\right) \\
& -\frac{\left(\alpha_{y} \sinh \left(\beta_{y} \eta\right) \sin \left(\alpha_{y}\right)\right)}{\beta_{y}} \\
& \left.\left.+\frac{\left(\beta_{y} \sin \left(\alpha_{y} \eta\right) \sinh \left(\beta_{y}\right)\right)}{\alpha_{y}}\right]\right\}\left\{\alpha _ { x } \beta _ { x } \left[2 \cos \left(\alpha_{x}\right)\right.\right. \\
& \cdot \cosh \left(\beta_{x}\right)+\frac{\left(\sin \left(\alpha_{x}\right) \sinh \left(\beta_{x}\right)\left(\alpha_{x}{ }^{2}-\beta_{x}{ }^{2}\right)\right)}{\left(\alpha_{x} \beta_{x}\right)} \\
& -2]\left[2 \cos \left(\alpha_{y}\right) \cosh \left(\beta_{y}\right)\right. \\
& \left.\left.+\frac{\left(\sin \left(\alpha_{y}\right) \sinh \left(\beta_{y}\right)\left(\alpha_{y}{ }^{2}-\beta_{y}{ }^{2}\right)\right)}{\left(\alpha_{y} \beta_{y}\right)}-2\right]\right\}^{-1}, \\
& N_{11 f}=\left\{\left[\beta _ { y } \left(\sin \left(\alpha_{y}(\eta-1)\right)\right.\right.\right. \\
& \left.+\cosh \left(\beta_{y} \eta\right) \sin \left(\alpha_{y}\right)-\sin \left(\alpha_{y} \eta\right) \cosh \left(\beta_{y}\right)\right) \\
& +\alpha_{y}\left(\sinh \left(\beta_{y}(\eta-1)\right)+\cos \left(\alpha_{y} \eta\right) \sinh \left(\beta_{y}\right)\right. \\
& \left.\left.-\sinh \left(\beta_{y} \eta\right) \cos \left(\alpha_{y}\right)\right)\right]\left[\cos \left(\alpha_{x}(\xi-1)\right)\right. \\
& +\cosh \left(\beta_{x}(\xi-1)\right)-\cos \left(\alpha_{x} \xi\right) \cosh \left(\beta_{x}\right) \\
& -\cosh \left(\beta_{x} \xi\right) \cos \left(\alpha_{x}\right)-\frac{\left(\alpha_{x} \sinh \left(\beta_{x} \xi\right) \sin \left(\alpha_{x}\right)\right)}{\beta_{x}} \\
& \left.\left.+\frac{\left(\beta_{x} \sin \left(\alpha_{x} \xi\right) \sinh \left(\beta_{x}\right)\right)}{\alpha_{x}}\right]\right\}\left\{\alpha _ { y } \beta _ { y } \left[2 \cos \left(\alpha_{x}\right)\right.\right. \\
& \cdot \cosh \left(\beta_{x}\right)+\frac{\left(\sin \left(\alpha_{x}\right) \sinh \left(\beta_{x}\right)\left(\alpha_{x}^{2}-\beta_{x}^{2}\right)\right)}{\left(\alpha_{x} \beta_{x}\right)} \\
& -2]\left[2 \cos \left(\alpha_{y}\right) \cosh \left(\beta_{y}\right)\right. \\
& \left.\left.+\frac{\left(\sin \left(\alpha_{y}\right) \sinh \left(\beta_{y}\right)\left(\alpha_{y}{ }^{2}-\beta_{y}{ }^{2}\right)\right)}{\left(\alpha_{y} \beta_{y}\right)}-2\right]\right\}^{-1},
\end{aligned}
$$




$$
\begin{aligned}
& N_{12 f}=\left\{\left[\beta _ { y } \left(\sin \left(\alpha_{y}(\eta-1)\right)+\cosh \left(\beta_{y} \eta\right) \sin \left(\alpha_{y}\right)\right.\right.\right. \\
& \left.-\sin \left(\alpha_{y} \eta\right) \cosh \left(\beta_{y}\right)\right)+\alpha_{y}\left(\sinh \left(\beta_{y}(\eta-1)\right)\right. \\
& \left.\left.+\cos \left(\alpha_{y} \eta\right) \sinh \left(\beta_{y}\right)-\sinh \left(\beta_{y} \eta\right) \cos \left(\alpha_{y}\right)\right)\right] \\
& \cdot\left[\beta _ { x } \left(\sin \left(\alpha_{x}(\xi-1)\right)+\cosh \left(\beta_{x} \xi\right) \sin \left(\alpha_{x}\right)\right.\right. \\
& \left.-\sin \left(\alpha_{x} \xi\right) \cosh \left(\beta_{x}\right)\right)+\alpha_{x}\left(\sinh \left(\beta_{x}(\xi-1)\right)\right. \\
& \left.\left.\left.+\cos \left(\alpha_{x} \xi\right) \sinh \left(\beta_{x}\right)-\sinh \left(\beta_{x} \xi\right) \cdot \cos \left(\alpha_{x}\right)\right)\right]\right\} \\
& \left\{\alpha _ { x } \alpha _ { y } \beta _ { x } \beta _ { y } \left[2 \cos \left(\alpha_{x}\right) \cosh \left(\beta_{x}\right)\right.\right. \\
& \left.+\frac{\left(\sin \left(\alpha_{x}\right) \sinh \left(\beta_{x}\right)\left(\alpha_{x}{ }^{2}-\beta_{x}{ }^{2}\right)\right)}{\left(\alpha_{x} \beta_{x}\right)}-2\right][2 \\
& \cdot \cos \left(\alpha_{y}\right) \cosh \left(\beta_{y}\right) \\
& \left.\left.+\frac{\left(\sin \left(\alpha_{y}\right) \sinh \left(\beta_{y}\right)\left(\alpha_{y}{ }^{2}-\beta_{y}{ }^{2}\right)\right)}{\left(\alpha_{y} \beta_{y}\right)}-2\right]\right\}^{-1}, \\
& N_{13 f}=\left\{\left[\cos \left(\alpha_{x} \xi\right)+\cosh \left(\beta_{x} \xi\right)-\cos \left(\alpha_{x}\right)\right.\right. \\
& \cdot \cosh \left(\beta_{x}(\xi-1)\right)-\cosh \left(\beta_{x}\right) \cos \left(\alpha_{x}(\xi-1)\right) \\
& +\frac{\left(\alpha_{x} \sin \left(\alpha_{x}\right) \sinh \left(\beta_{x}(\xi-1)\right)\right)}{\beta_{x}} \\
& \left.-\frac{\left(\beta_{x} \sinh \left(\beta_{x}\right) \sin \left(\alpha_{x}(\xi-1)\right)\right)}{\alpha_{x}}\right] \\
& \cdot\left[\cos \left(\alpha_{y}(\eta-1)\right)+\cosh \left(\beta_{y}(\eta-1)\right)\right. \\
& -\cos \left(\alpha_{y} \eta\right) \cosh \left(\beta_{y}\right)-\cosh \left(\beta_{y} \eta\right) \cos \left(\alpha_{y}\right) \\
& -\frac{\left(\alpha_{y} \sinh \left(\beta_{y} \eta\right) \sin \left(\alpha_{y}\right)\right)}{\beta_{y}} \\
& \left.\left.+\frac{\left(\beta_{y} \sin \left(\alpha_{y} \eta\right) \sinh \left(\beta_{y}\right)\right)}{\alpha_{y}}\right]\right\}\left\{\left[2 \cos \left(\alpha_{x}\right)\right.\right. \\
& \cdot \cosh \left(\beta_{x}\right)+\frac{\left(\sin \left(\alpha_{x}\right) \sinh \left(\beta_{x}\right)\left(\alpha_{x}^{2}-\beta_{x}^{2}\right)\right)}{\left(\alpha_{x} \beta_{x}\right)} \\
& -2]\left[2 \cos \left(\alpha_{y}\right) \cosh \left(\beta_{y}\right)\right. \\
& \left.\left.+\frac{\left(\sin \left(\alpha_{y}\right) \sinh \left(\beta_{y}\right)\left(\alpha_{y}{ }^{2}-\beta_{y}{ }^{2}\right)\right)}{\left(\alpha_{y} \beta_{y}\right)}-2\right]\right\}^{-1} \text {, } \\
& \begin{aligned}
N_{14 f} & =-\left\{\left[\beta _ { x } \left(\cosh \left(\beta_{x}\right) \sin \left(\alpha_{x}(\xi-1)\right)\right.\right.\right. \\
- & \left.\sin \left(\alpha_{x} \xi\right)+\sin \left(\alpha_{x}\right) \cosh \left(\beta_{x}(\xi-1)\right)\right) \\
+ & \alpha_{x}\left(\cos \left(\alpha_{x}\right) \sinh \left(\beta_{x}(\xi-1)\right)-\sinh \left(\beta_{x} \xi\right)\right. \\
+ & \left.\left.\sinh \left(\beta_{x}\right) \cos \left(\alpha_{x}(\xi-1)\right)\right)\right]\left[\cos \left(\alpha_{y}(\eta-1)\right)\right. \\
+ & \cosh \left(\beta_{y}(\eta-1)\right)-\cos \left(\alpha_{y} \eta\right) \cosh \left(\beta_{y}\right) \\
- & \cosh \left(\beta_{y} \eta\right) \cos \left(\alpha_{y}\right) \\
& \left.\left.-\frac{\left(\alpha_{y} \sinh \left(\beta_{y} \eta\right) \sin \left(\alpha_{y}\right)\right)}{\beta_{y}}\right]\right\}\left\{\alpha _ { x } \beta _ { x } \left[2 \cos \left(\alpha_{x}\right)\right.\right. \\
+ & \frac{\left(\beta_{y} \sin \left(\alpha_{y} \eta\right) \sinh \left(\beta_{y}\right)\right)}{\alpha_{y}}\left(\alpha_{x} \beta_{x}\right) \\
+ & \cosh \left(\beta_{x}\right)+\frac{\left(\sin \left(\alpha_{x}\right) \sinh \left(\beta_{x}\right)\left(\alpha_{x}^{2}-\beta_{x}^{2}\right)\right)}{\left(\alpha_{y} \beta_{y}\right)} \\
+ & 2]\left[2 \cos \left(\alpha_{y}\right) \cosh \left(\beta_{y}\right)\right. \\
+ & \frac{\left(\sin \left(\alpha_{y}\right) \sinh \left(\beta_{y}\right)\left(\alpha_{y}{ }^{2}-\beta_{y}{ }^{2}\right)\right)}{\left.\left.{ }^{2}-2\right]\right\}},
\end{aligned} \\
& N_{15 f}=\left\{\left[\beta _ { y } \left(\sin \left(\alpha_{y}(\eta-1)\right)\right.\right.\right. \\
& \left.+\cosh \left(\beta_{y} \eta\right) \sin \left(\alpha_{y}\right)-\sin \left(\alpha_{y} \eta\right) \cosh \left(\beta_{y}\right)\right) \\
& +\alpha_{y}\left(\sinh \left(\beta_{y}(\eta-1)\right)+\cos \left(\alpha_{y} \eta\right) \sinh \left(\beta_{y}\right)\right. \\
& \left.\left.-\sinh \left(\beta_{y} \eta\right) \cos \left(\alpha_{y}\right)\right)\right]\left[\cos \left(\alpha_{x} \xi\right)+\cosh \left(\beta_{x} \xi\right)\right. \\
& -\cos \left(\alpha_{x}\right) \cosh \left(\beta_{x}(\xi-1)\right)-\cosh \left(\beta_{x}\right) \\
& \cdot \cos \left(\alpha_{x}(\xi-1)\right) \\
& +\frac{\left(\alpha_{x} \sin \left(\alpha_{x}\right) \sinh \left(\beta_{x}(\xi-1)\right)\right)}{\beta_{x}} \\
& \left.\left.-\frac{\left(\beta_{x} \sinh \left(\beta_{x}\right) \sin \left(\alpha_{x}(\xi-1)\right)\right)}{\alpha_{x}}\right]\right\}\left\{\alpha_{y} \beta_{y}[2\right. \\
& \cdot \cos \left(\alpha_{x}\right) \cosh \left(\beta_{x}\right) \\
& \left.+\frac{\left(\sin \left(\alpha_{x}\right) \sinh \left(\beta_{x}\right)\left(\alpha_{x}^{2}-\beta_{x}^{2}\right)\right)}{\left(\alpha_{x} \beta_{x}\right)}-2\right][2 \\
& \cdot \cos \left(\alpha_{y}\right) \cosh \left(\beta_{y}\right)
\end{aligned}
$$




$$
\begin{aligned}
+ & \left.\left.\frac{\left(\sin \left(\alpha_{y}\right) \sinh \left(\beta_{y}\right)\left(\alpha_{y}{ }^{2}-\beta_{y}{ }^{2}\right)\right)}{\left(\alpha_{y} \beta_{y}\right)}-2\right]\right\}^{-1}, \\
N_{16 f}=-\left\{\left[\beta _ { x } \left(\cosh \left(\beta_{x}\right) \sin \left(\alpha_{x}(\xi-1)\right)\right.\right.\right. & \left.-\sin \left(\alpha_{x} \xi\right)+\sin \left(\alpha_{x}\right) \cosh \left(\beta_{x}(\xi-1)\right)\right) \\
+ & \alpha_{x}\left(\cos \left(\alpha_{x}\right) \sinh \left(\beta_{x}(\xi-1)\right)-\sinh \left(\beta_{x} \xi\right)\right. \\
+ & \left.\left.\sinh \left(\beta_{x}\right) \cos \left(\alpha_{x}(\xi-1)\right)\right)\right] \\
& +\left[\beta _ { y } \left(\sin \left(\alpha_{y}(\eta-1)\right)+\cosh \left(\beta_{y} \eta\right) \sin \left(\alpha_{y}\right)\right.\right. \\
& \left.-\sin \left(\alpha_{y} \eta\right) \cosh \left(\beta_{y}\right)\right)+\alpha_{y}\left(\sinh \left(\beta_{y}(\eta-1)\right)\right. \\
+ & \left.\left.\left.\cos \left(\alpha_{y} \eta\right) \sinh \left(\beta_{y}\right)-\sinh \left(\beta_{y} \eta\right) \cdot \cos \left(\alpha_{y}\right)\right)\right]\right\} \\
& +\left\{\alpha _ { x } \alpha _ { y } \beta _ { x } \beta _ { y } \left[2 \cos \left(\alpha_{x}\right) \cosh \left(\beta_{x}\right)\right.\right. \\
& \left.+\frac{\left(\sin \left(\alpha_{x}\right) \sinh \left(\beta_{x}\right)\left(\alpha_{x}{ }^{2}-\beta_{x}{ }^{2}\right)\right)}{\left(\alpha_{x} \beta_{x}\right)}-2\right][2 \\
+ & \cos \left(\alpha_{y}\right) \cosh \left(\beta_{y}\right) \\
+ & \left.\left.\frac{\left(\sin \left(\alpha_{y}\right) \sinh \left(\beta_{y}\right)\left(\alpha_{y}{ }^{2}-\beta_{y}{ }^{2}\right)\right)}{\left(\alpha_{y} \beta_{y}\right)}-2\right]\right\}^{-1} .
\end{aligned}
$$

\section{Disclosure}

This paper presents the results of a recent research, conducted by the first author under the supervision of the second author.

\section{Conflicts of Interest}

The authors declare that there are no conflicts of interest regarding the publication of this paper.

\section{Acknowledgments}

The authors wish to acknowledge the support provided by NSERC, OGS, and Ryerson University.

\section{References}

[1] C. L. M. H. Navier, "Bulletin des Sciences de la Societe Philomathique de Paris," 1823.

[2] M. Levy, "Memoire sur la theorie des plaques elastiques planes," Journal de Mathématiques Pures et Appliquées, vol. 3, p. 219, 1899.

[3] M. Levy, "Sur L'equilibre elasticque d'une Plaque Rectangulaire," Comptes Rendus de l'Académie des Sciences, vol. 129, pp. 535-539, 1899.
[4] G. Jin et al., Structural Vibration: A Uniform Accurate Solution for Laminated Beams, Plates and Shells with General Boundary Conditions, Springer Heidelberg, New York, NY, USA, 2015.

[5] Q. Wang, D. Shi, Q. Liang, and F. Ahad, "An improved Fourier series solution for the dynamic analysis of laminated composite annular, circular, and sector plate with general boundary conditions," Journal of Composite Materials, vol. 50, no. 30, pp. 41994233, 2016.

[6] M. Boscolo and J. R. Banerjee, "Dynamic stiffness formulation for plates using first order shear deformation theory," in Proceedings of the 51st AIAA/ASME/ASCE/AHS/ASC Structures, Structural Dynamics and Materials Conference, Orlando, Florida, USA, 2010.

[7] M. Boscolo and J. R. Banerjee, "Dynamic stiffness method for exact inplane free vibration analysis of plates and plate assemblies," Journal of Sound and Vibration, vol. 330, no. 12, pp. 2928-2936, 2011.

[8] X. Liu and J. R. Banerjee, "An exact spectral-dynamic stiffness method for free flexural vibration analysis of orthotropic composite plate assemblies - Part I: Theory," Composite Structures, vol. 132, pp. 1274-1287, 2015.

[9] X. Liu and J. R. Banerjee, "An exact spectral-dynamic stiffness method for free flexural vibration analysis of orthotropic composite plate assemblies - Part II: Applications," Composite Structures, vol. 132, pp. 1288-1302, 2015.

[10] X. Liu and J. R. Banerjee, "Free vibration analysis for plates with arbitrary boundary conditions using a novel spectral-dynamic stiffness method," Computers \& Structures, vol. 164, pp. 108-126, 2016.

[11] K. H. Huebner et al., The Finite Element Method for Engineers, John Wiley and Sons Inc, New York, NY, USA, 2001.

[12] R. Bellman and J. Casti, "Differential quadrature and long-term integration," Journal of Mathematical Analysis and Applications, vol. 34, pp. 235-238, 1971.

[13] R. Bellman, B. G. Kashef, and J. Casti, "Differential quadrature: a technique for the rapid solution of nonlinear partial differential equations," Journal of Computational Physics, vol. 10, pp. 40-52, 1972.

[14] C. Shu, "Application of differential quadrature method to structural and vibration analysis," in Differential Quadrature and Its Application in Engineering, Springer, London, UK, 2000.

[15] S. A. Eftekhari and A. A. Jafari, "A mixed method for free and forced vibration of rectangular plates," Applied Mathematical Modelling, vol. 36, no. 6, pp. 2814-2831, 2012.

[16] P. Singhal and G. Bindal, "Generalised Differential Quadrature Method in the Study of Free Vibration Analysis of Monoclinic Rectangular Plates," American Journal of Computational and Applied Mathematics, vol. 2, no. 4, pp. 166-173, 2012.

[17] S. M. Hashemi, Free-vibrational analysis of rotating beam-like structures: A dynamic finite element approach [Ph.D. thesis], Laval University, Quebec, Canada, 1998.

[18] S. M. Hashemi and M. J. Richard, "A Dynamic Finite Element (DFE) method for free vibrations of bending-torsion coupled beams," Aerospace Science and Technology, vol. 4, no. 1, pp. 41$55,2000$.

[19] S. M. Hashemi and E. J. Adique, "A quasi-exact dynamic finite element for free vibration analysis of sandwich beams," Applied Composite Materials, vol. 17, no. 2, pp. 259-269, 2010.

[20] M. T. Kashani, S. Jayasinghe, and S. M. Hashemi, "Dynamic finite element analysis of bending-torsion coupled beams subjected to combined axial load and end moment," Shock and Vibration, vol. 2015, Article ID 471270, 12 pages, 2015. 
[21] S. Jayasinghe and S. M. Hashemi, "Frequency dependant trigonometric shape functions for thin rectangular plate elements," in Proceedings of the 23rd International Congress on Sound and Vibration (ICSV'16), 2016.

[22] M. A. Bhatti, Advanced Topics in Finite Element Analysis of Structures, John Wiley and Sons Inc, Hoboken, NJ, USA, 2006. 


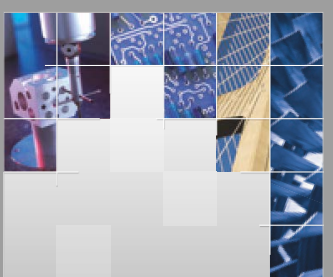

\section{Enfincering}
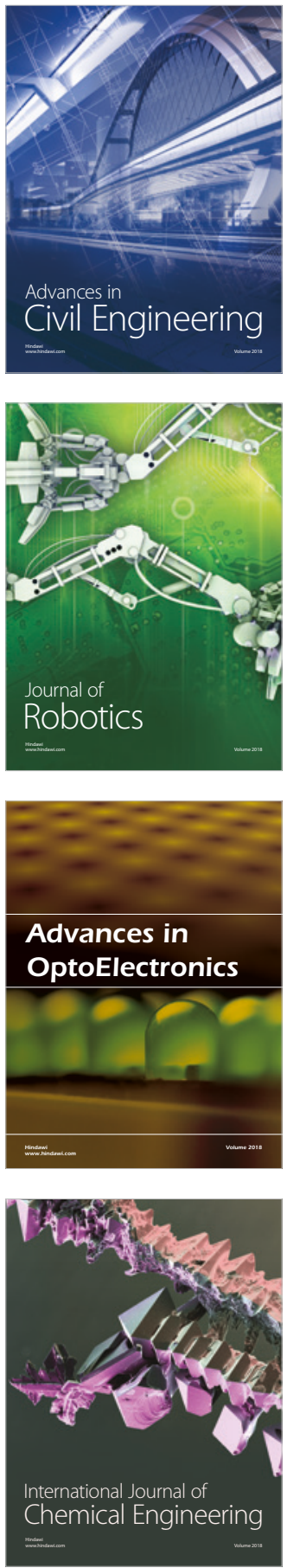

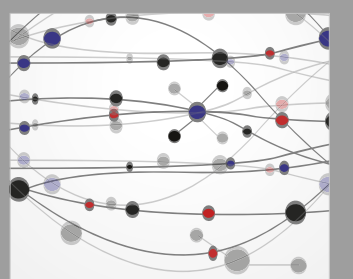

\section{Rotating \\ Machinery}

The Scientific World Journal

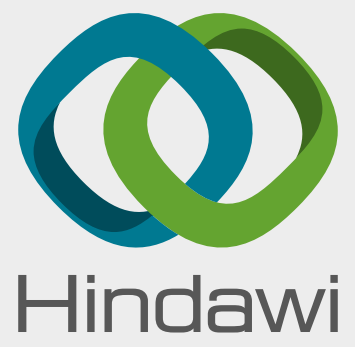

Submit your manuscripts at

www.hindawi.com
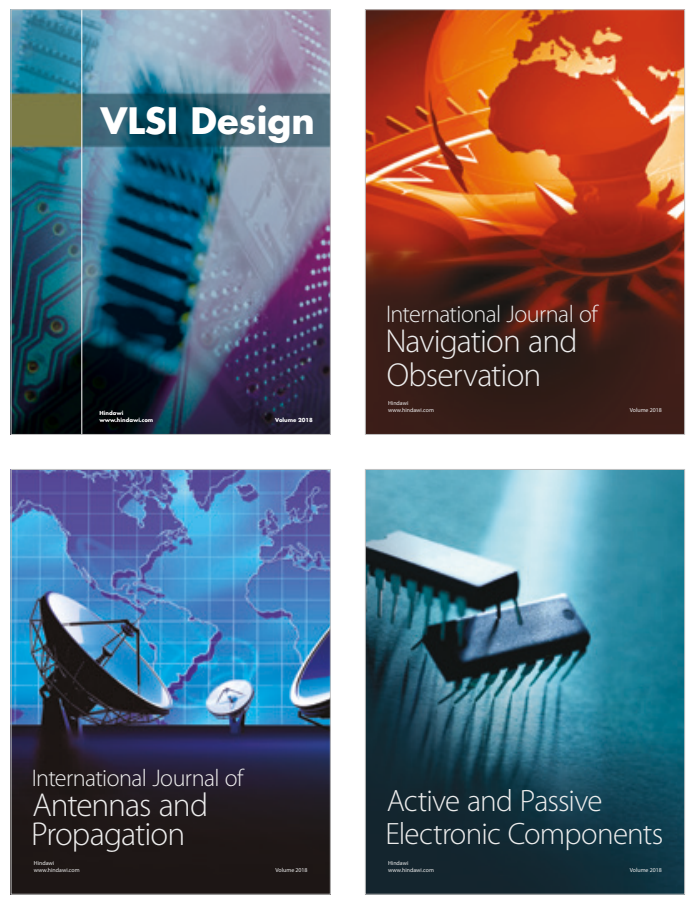
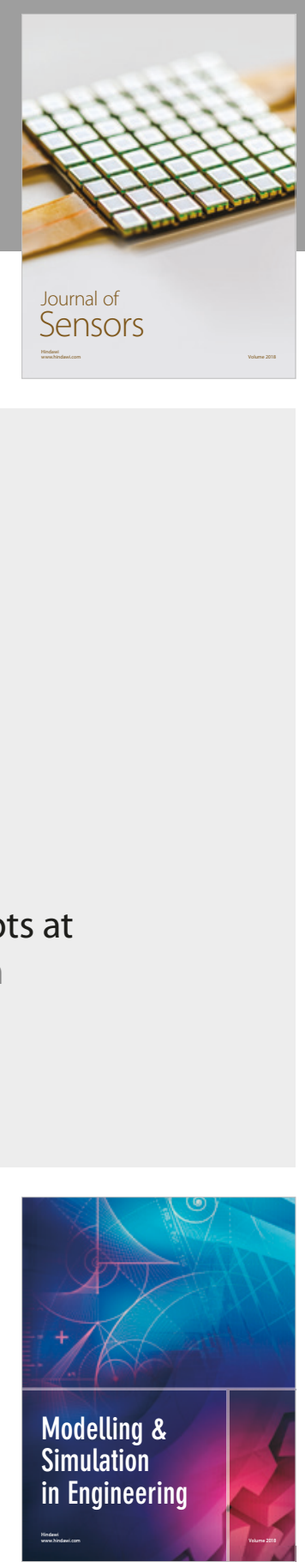

\section{Advances \\ Multimedia}
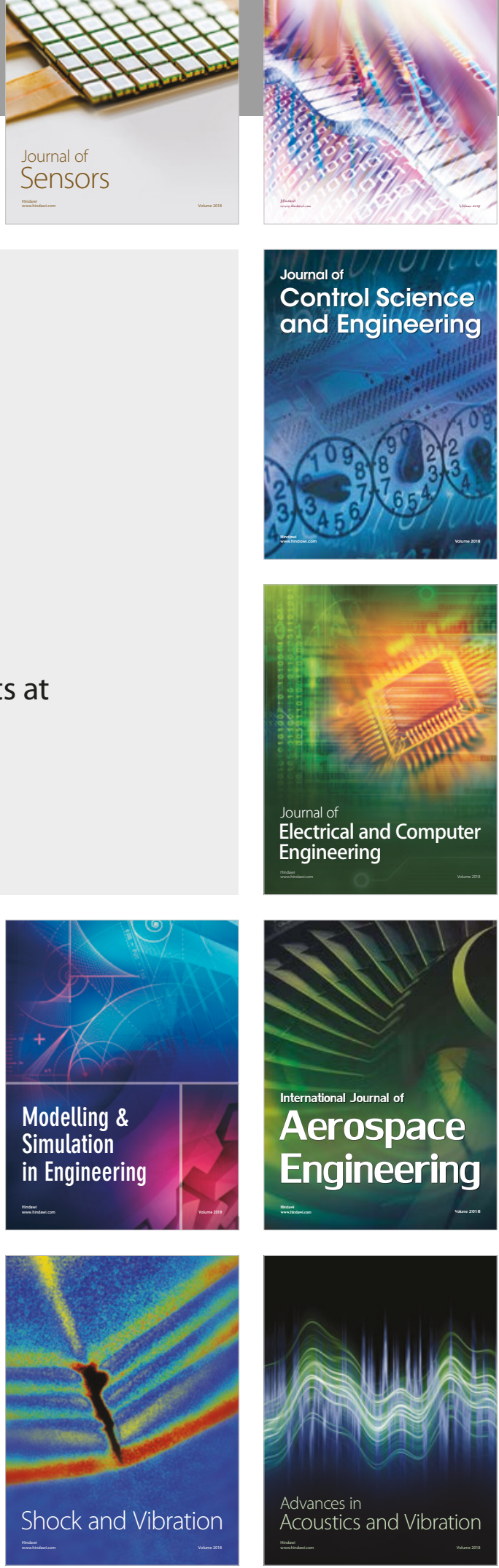